\title{
Multi-instanton contributions to anomalous quark interactions
}

\author{
Robert D. Pisarski@* and Fabian Rennecke $\odot^{\dagger}$ \\ Department of Physics, Brookhaven National Laboratory, Upton, New York 11973, USA
}

(Received 5 December 2019; accepted 3 June 2020; published 19 June 2020)

\begin{abstract}
In quantum chromodynamics $(\mathrm{QCD})$, the role which topologically nontrivial configurations play in splitting the singlet pseudo-Goldstone meson, the $\eta^{\prime}$, from the octet is familiar. In addition, such configurations contribute to other processes which violate the axial $U(1)_{A}$ symmetry. While the nature of topological fluctuations in the vacuum is still unsettled, at temperatures above that for the chiral phase transition, they can be described by a dilute gas of instantons. We show that instantons of arbitrary topological charge $Q$ generate anomalous interactions between $2 N_{f}|Q|$ quarks, which for $Q=1$ make the $\eta^{\prime}$ heavy. For two flavors we compute an anomalous quartic meson coupling and discuss its implications for the phenomenology of the chiral phase transition. A dilute instanton gas suggests that for cold, dense quarks, instantons do not evaporate until very high densities, when the baryon chemical potential is $\gtrsim 2 \mathrm{GeV}$.
\end{abstract}

DOI: 10.1103/PhysRevD.101.114019

\section{INTRODUCTIONS}

In quantum chromodynamics (QCD), the up, down and strange quarks are relatively light, and there is an approximate global flavor symmetry of $S U(3)_{L} \times S U(3)_{R} \times$ $U(1)_{A}$. When the hadronic vacuum spontaneously breaks chiral symmetry, a flavor octet of light pseudo-Goldstone bosons is generated, which are the $\pi, K$, and $\eta$ mesons of broken $S U(3)_{L} \times S U(3)_{R}$. When QCD first emerged, it was a puzzle why there is not an associated ninth pseudoGoldstone boson in the flavor singlet channel, the $\eta^{\prime}$, from the breaking of the axial $U(1)_{A}$ symmetry.

This occurs because while classically there is an axial $U(1)_{A}$ symmetry, it is not valid quantum mechanically because of an anomaly [1]. There are topologically nontrivial fluctuations which violate the $U(1)_{A}$ symmetry [2] and make the $\eta^{\prime}$ heavy [3]. Classically these configurations are instantons: these have a topological winding number equal to an integer $Q$, and an (Euclidean) action equal to $8 \pi^{2}|Q| / g^{2}$, where $g$ is the coupling constant of QCD [4-39]. Instantons split the singlet $\eta^{\prime}$ from the octet of pseudo-Goldstone bosons and also generate the $\theta$ parameter of QCD [5].

There are several open questions regarding the nature of topological fluctuations in the QCD vacuum. In absence of

\footnotetext{
pisarski@bnl.gov

frennecke@bnl.gov
}

Published by the American Physical Society under the terms of the Creative Commons Attribution 4.0 International license. Further distribution of this work must maintain attribution to the author(s) and the published article's title, journal citation, and DOI. Funded by SCOAP. a large energy scale to cut off the size of the instantons, their fluctuations on any length scale become relevant, and the integration over their contribution blows up. This is cured nonperturbatively through confinement, where dense topologically nontrivial fluctuations may form an instanton liquid [16-18]. Furthermore, it is expected that QCD behaves smoothly as the number of colors, $N_{c}$, goes to infinity $[40,41]$. In this limit, the contribution of a single instanton vanishes exponentially, while current algebra can be used to show that the $\eta^{\prime}$ is still split from the octet of pseudo-Goldstone bosons [40]. This could occur if there are topologically nontrivial fluctuations whose topological charge is not an integer, but an integer times $1 / N_{c}$; in certain limits, such as for adjoint QCD on a femto-torus, this can be shown semiclassically [30,31].

However, if the effective coupling is small, e.g., at high temperature or quark density, then a semiclassical analysis is valid, and topologically nontrivial fluctuations can be approximated as a dilute instanton gas [12,13]. Numerical simulations of lattice QCD provide insight into how the topological structure changes with temperature [32-39]. Remarkably, these demonstrate that the overall power of the topological susceptibility with respect to the temperature $T$ is given by a dilute instanton gas above temperatures as low as a few hundred $\mathrm{MeV}$ [34-37].

In this paper we address a modest problem and consider quantities which are nonzero only because of topologically nontrivial configurations, using a dilute instanton gas as an illustrative example. Studies of the phenomenological implications of the axial anomaly, including the effects mentioned above, have been based on effective quark interactions that are generated in a dilute gas of instantons of unit topological charge [4]. Here we generalize this by 
demonstrating that effective $2 N_{f}|Q|$-quark interactions are generated in a dilute gas of instantons of arbitrary topological charge $Q$ [7-10]. Even though semiclassically such topological field configurations are suppressed exponentially, these interactions can give rise to novel anomalous effects related uniquely to fluctuations of higher topological charge. We develop a general formalism for including the effects of higher topological charge and use this to explicitly work out the local effective interaction for $Q= \pm 2$, for the case where the constituent-instantons, which we define before Eq. (1), are small. At low energies and for two quark flavors this is a quartic meson interaction. In the third section we present a phenomenological analysis, analyzing the effects of anomalous meson interactions in mean field theory and use a dilute instanton gas to roughly estimate their magnitude. Technical details of the computation are given in the Appendix.

\section{MULTI-INSTANTON-INDUCED INTERACTIONS}

We start with an analysis for arbitrary topological charge, generalizing that of 't Hooft [4]. We consider the generating functional of QCD for Gaussian fluctuations around a background of instantons with topological charge $Q$, which we term $Q$-instantons. For a $Q$-(anti-) instanton background, massless quarks have $N_{f}|Q|$ (right-) left-handed zero modes [6]. We show that the functional zero mode determinant of quarks has the structure of a $2 N_{f}|Q|$-quark correlation function and compute its coupling constant in a dilute gas of $Q$-instantons.

The zero modes of gauge fields arise from symmetries, such as translations, that yield inequivalent instanton solutions. This defines a moduli space which is parametrized by the collective coordinates of the instantons. The general $Q$-instanton has been constructed by Atiyah et al. (ADHM) [8-10]. It can be viewed as a superposition of $Q$ individual instantons with unit charge, where each constituent is described by a location $z_{i}$, a size $\rho_{i}$ and an orientation in the gauge group $U_{i}$. There are then $4 N_{c}$ collective coordinates for each constituent-instanton, so the moduli space of the $Q$-instanton has dimension $4 N_{c}|Q|$. Schematically, the generating functional is

$$
\begin{aligned}
Z^{(Q)}[J] & =\int \mathcal{D} \chi \exp \left\{-S\left[\chi+\chi^{(Q)}\right]+\int_{x} \bar{\psi} J \psi\right\} \\
& \approx \int d C_{Q} n_{Q}\left(C_{Q}\right) \operatorname{det}_{0}(J)
\end{aligned}
$$

where $\chi=\left(A_{\mu}, c, \bar{c}, \psi, \bar{\psi}\right)$ contains the fluctuating gluon, ghost and quark fields, and $\chi^{(Q)}=\left(A_{\mu}^{(Q)}, 0,0,0,0\right)$ contains the $Q$-instanton background field $A_{\mu}^{(Q)}$; to avoid notational clutter, the superscript $Q$ in $A_{\mu}^{(Q)}$ denotes the topological charge. $S[\chi]$ is the gauge-fixed action of QCD in Euclidean spacetime. In the second line we integrate the path integral over the nonzero modes to leading order in the saddle point approximation, leaving only the integration over the collective coordinates $C_{Q}$. The instanton density $n_{Q}$ contains the functional determinants of the zero and nonzero modes of gluons and ghosts, the nonzero mode determinant of the quarks and the Jacobian from changing the integration over zero modes to collective coordinates [42].

Our main ingredient is the zero modes of massless quarks [11]. Due to the axial anomaly, the Dirac operator in the presence of the $Q$-instanton, $D^{(Q)}=\gamma_{\mu}\left(\partial_{\mu}+A_{\mu}^{(Q)}\right)$, has $N_{f}|Q|$ zero modes, $D^{(Q)} \psi_{f i}^{(Q)}=0$, where $f=1, \ldots, N_{f}$ is an index for flavor and $i=1, \ldots,|Q|$ is a topological charge index. Because of the zero modes, the generating functional is only nonzero in the presence of a source $J$, which generates the quark zero mode determinant, $\operatorname{det}_{0}(J)$, in Eq. (1).

The generating functional in Eq. (1) has first been computed for $Q=1$ and $N_{c}=2$ [4] and arbitrary $N_{c}$ [43]. For $|Q|>1$, the generating functional to one loop order is only known in certain limits [20].

One limit where one can compute is when the distance between the locations of the constituent-instantons are much larger than their sizes; i.e., $\left|R_{i j}\right| \equiv\left|z_{i}-z_{j}\right| \gg \rho_{i}$ for all $i \neq j$. In this case, at leading order, the $Q$-instanton can be viewed as $|Q|$ instantons of unit charge which are well separated. Expanding the general ADHM-solution in this small limit, up to order $\sim(\rho /|R|)^{4}$ the path integral factorizes into a product of constituent-instanton contributions [10],

$$
Z^{(Q)}[J] \rightarrow \int \frac{\left[d C_{1} n_{1}\left(C_{1}\right)\right]^{(Q)}}{Q !} \operatorname{det}_{0}(J) .
$$

For ease of notation, we assume $Q>0$ as anti-instantons with $Q<0$ can be treated similarly. The factor of $Q$ ! arises because the constituent-instantons can be treated as identical particles. The collective coordinate measure for the $i$ th constituent-instanton is $d C_{i}=d \rho_{i} d^{4} z_{i} d U_{i} . d U_{i}$ is the Haar measure of the coset space $S U\left(N_{c}\right) / \mathcal{I}_{N_{c}}$, where the stability group of the instanton $\mathcal{I}_{N_{c}}$ is given by all $S U\left(N_{c}\right)$-transformations that leave the instanton unchanged. We emphasize that in the small limit the instanton density only depends upon the sizes $\rho_{i}$.

Using the methods of Ref. [11], for small constituentinstantons, to $\sim(\rho /|R|)^{3}$, the zero modes of a $Q$-instanton are simply given by the corresponding zero modes for $Q=1$, and so the quark zero mode determinant factorizes, $Z^{(Q)}[J]=\left(Z^{(1)}[J]\right)^{Q} / Q$ !. Thus, for a dilute gas of $Q$-instantons, the effective Lagrangian which results is the $Q^{\text {th }}$ power of the 't Hooft determinant, where each determinant is integrated over space-time, $\sim\left[\int d^{4} x \operatorname{det}\left(\bar{\psi}_{L}(x) \psi_{R}(x)\right)\right]^{Q}$. Due to the complete factorization of the generating functional 
into single-instanton contributions to order $(\rho /|R|)^{3}$ in the limit of small constituent-instantons, this is fully described by a dilute gas of single-instantons.

In addition, there are genuine multi-instanton contributions which cannot be described by single-instantons. They give rise to a local interaction which is given by a single integral over space-time for the $Q^{\text {th }}$ power of the 't Hooft determinant, $\sim \int d^{4} x\left[\operatorname{det}\left(\bar{\psi}_{L}(x) \psi_{R}(x)\right)\right]^{Q}$. To find this, one needs to account for the overlap between the constituentinstantons. The result of a novel and lengthy analysis, given in the Appendix, shows that to order $\sim(\rho /|R|)^{4}$ the only change we need to account for is the difference in the quark zero modes. The zero mode for the $Q=1$ instanton is

$$
\psi_{f i}\left(x, z_{i}\right)=\frac{\sqrt{2}}{\pi} \frac{U_{i} \rho_{i}}{\left[\left(x-z_{i}\right)^{2}+\rho_{i}^{2}\right]^{3 / 2}} \frac{\gamma_{\mu}\left(x-z_{i}\right)_{\mu}}{\left|x-z_{i}\right|} \varphi_{R},
$$

where $\varphi_{R}$ is a right-handed spinor so that the zero mode is left-handed. It will be useful later to note that far from the instanton the quark zero mode is proportional to the free quark propagator $\Delta(x)=\gamma_{\mu} x_{\mu} / 2 \pi^{2}\left(x^{2}\right)^{2}$.

For simplicity we consider instantons with charge two. Using the methods of Ref. [11] to derive the quark zero modes from the ADHM-solution in the limit of small constituent-instantons, the $2 N_{f}$ zero modes for $Q=2$ can be expressed in terms of the $Q=1$ zero modes as

$$
\psi_{f 1}^{(2)}=\psi_{f 1}-\mathbb{X}_{1} \hat{\psi}_{f 2}, \quad \psi_{f 2}^{(2)}=\psi_{f 2}-\mathbb{X}_{2} \hat{\psi}_{f 1},
$$

where $\hat{\psi}_{f i}=U_{j} U_{i}^{\dagger} \psi_{f i}$ and

$$
\mathbb{X}_{i}\left(x, z_{i}\right)=\frac{\rho_{1} \rho_{2}\left|x-z_{i}\right|}{\left[\left(x-z_{i}\right)^{2}+\rho_{i}^{2}\right]^{3 / 2}} .
$$

So for small constituent-instantons the $Q=2$ zero modes decompose into separate $Q=1$ zero modes, connected by the overlap term $\mathbb{X}_{i}$.

In general, the determinant depends on the locations of the constituent-instantons, $z_{1}$ and $z_{2}$, which can be rewritten as an average position $z=\left(z_{1}+z_{2}\right) / 2$ and their separation, $R_{12}$. Integrating over $R_{12}$ the zero mode determinant becomes

$$
\operatorname{det}_{0}(J) \propto \mathcal{I}_{N_{f}} \prod_{i=1}^{2} \int_{U_{i}} \prod_{f=1}^{N_{f}} \int_{x_{f i}} \psi_{f i}^{\dagger}\left(x_{f i}, z\right) J\left(x_{f i}\right) \psi_{f i}\left(x_{f i}, z\right),
$$

where $\mathcal{I}_{N_{f}}$ measures the overlap of the zero modes,

$$
\mathcal{I}_{N_{f}}=\int d^{4} R_{12} \prod_{f} \mathbb{X}_{i}^{2}\left(x_{f i}, R_{12}\right) .
$$

For one flavor the overlap integral is infrared-divergent, requiring a cutoff for large distances $\left|x-z_{i}\right|$. Presumably, this cutoff is set by the average separation between an instanton and an anti-instanton. For two or more flavors, a local interaction is generated when all quark zero modes are close to the same constituent-instanton [44], and we find

$$
\mathcal{I}_{N_{f} \geq 2}=\pi^{2} \frac{\left(N_{f}+1\right) !\left(2 N_{f}-3\right) !}{\left(3 N_{f}-1\right) !} \rho_{1}^{2 N_{f}} \rho_{2}^{4-2 N_{f}} .
$$

Because zero modes approach free quark propagators at large distances, Eq. (3), the zero mode determinant in Eq. (6) has the form of a $2 N_{f} Q$-quark correlation function. Hence, in direct generalization of [4], the generating functional in the presence of small constituent-instantons gives rise to an effective interaction between $4 N_{f}$ quarks. Assuming that the topological fluctuations are described by a dilute gas of instantons, the contribution from dilute $Q=2$ instantons and anti-instantons generates an anomalous contribution to the local effective Lagrangian, as a product of operators which are color singlet [45],

$\Delta \mathcal{L}_{\text {eff }}^{(2)}=\frac{-\kappa_{2}}{K_{2, N_{f}}}\left(\left[\operatorname{det}_{f g}\left(\bar{\psi}_{f} \mathbb{P}_{R} \psi_{g}\right)\right]^{2}+\left[\operatorname{det}_{f g}\left(\bar{\psi}_{f} \mathbb{P}_{L} \psi_{g}\right)\right]^{2}\right)$,

where $\mathbb{P}_{R / L}=\left(1 \pm \gamma_{5}\right) / 2$ are the right-/left-handed projection operators and $K_{Q, N_{f}}=(Q !)^{N_{f}} /\left(Q N_{f}\right)$ ! is a combinatorial factor. The effective coupling in this semiclassical analysis is

$$
\kappa_{2}=\left(8 \pi^{2}\right)^{2 N_{f}} \int_{\rho_{1}} n_{1}\left(\rho_{1}\right) \rho_{1}^{5 N_{f}} \int_{\rho_{2}} n_{1}\left(\rho_{2}\right) \rho_{2}^{N_{f}} \mathcal{I}_{N_{f}} .
$$

This result generalizes the instanton-induced local interaction to topological charge $Q=2$. We note that, while the effective action induced by a single instanton breaks $U(1)_{A}$ down to the cyclic group $\mathbb{Z}_{N_{f}}$, the $Q=2$ contribution has a larger residual $\mathbb{Z}_{2 N_{f}}$-symmetry. The computation outlined here can be generalized to arbitrary topological charge.

\section{A LOW ENERGY MODEL}

Our analysis above applies only in the limit of a dilute instanton gas, as is applicable at high temperature [34-37] or density. We do expect that the anomalous quark interactions generated by higher topological charge are present at low temperature, where a dilute instanton gas is certainly not applicable. In this section, we first analyze the general effect of anomalous quark interactions, and then estimate their values in a dilute instanton gas. Thus these values should only be taken as an illustrative estimate of their overall magnitude and not as a definitive computation.

To illustrate the physical effect of interactions induced by higher topological charge, we consider a linear sigma model for $N_{f}=2$ that includes all anomalous interactions up to quartic order. These are generated in a dilute gas of 
instantons and anti-instantons with $Q=1$ and 2 . Classically, the global chiral symmetry of $\mathcal{G}_{\mathrm{cl}}=S U(2)_{L} \times$ $S U(2)_{R} \times U(1)_{A}$ is reduced to $\mathcal{G}_{\mathrm{A}}=S U(2)_{L} \times S U(2)_{R} \times$ $\mathbb{Z}_{N_{f}}$ by topological fluctuations. Effective mesons are given by $\Phi=(\sigma+i \eta)+\left(\vec{a}_{0}+i \vec{\pi}\right) \vec{\tau}$, with the Pauli-matrices $\vec{\tau}$. The resulting Lagrangian is a sum of two terms [24],

$$
\begin{aligned}
\mathcal{L}_{\mathcal{G}_{\mathrm{cl}}}= & \operatorname{tr}\left(\partial_{\mu} \Phi^{\dagger}\right)\left(\partial_{\mu} \Phi\right)+m^{2} \operatorname{Tr} \Phi^{\dagger} \Phi \\
& +\lambda_{1} \operatorname{Tr}\left(\Phi^{\dagger} \Phi\right)^{2}+\lambda_{2}\left(\operatorname{Tr} \Phi^{\dagger} \Phi\right)^{2}, \\
\mathcal{L}_{\mathcal{G}_{\mathrm{A}}}= & -\chi_{1}\left(\operatorname{det} \Phi+\operatorname{det} \Phi^{\dagger}\right) \\
& -\chi_{2}\left[(\operatorname{det} \Phi)^{2}+\left(\operatorname{det} \Phi^{\dagger}\right)^{2}\right] .
\end{aligned}
$$

We emphasize that taking into account the contributions from instantons and anti-instantons is necessary to ensure $\mathcal{C P}$-invariance. The term $\sim \chi_{1}$ arises from bosonizing the usual 't Hooft determinant from instantons with $Q= \pm 1$, while the term $\sim \chi_{2}$ is generated by bosonizing interactions with $Q= \pm 2$ in Eq. (9) [46].

We focus on the mass spectrum of mesons in the meanfield approximation. We use the $\sigma-, \eta-, a_{0}$-meson masses and $f_{\pi}$ to fix four of the five parameters of $\mathcal{L}$ in the vacuum. Chiral symmetry breaking is controlled by the mass parameter $m^{2}$. By varying $m^{2}$ relative to its vacuum value we define a reduced temperature $t=t\left(\mathrm{~m}^{2}\right)$, where $t=0$ corresponds to the vacuum and $t=1$ to the chiral phase transition. By choosing $\chi_{2}$ as a free parameter, we can study the impact of the topological charge-two term on the masses in the phases with broken and restored chiral symmetry. The resulting mass spectrum is shown in Fig. 1. The details of the computation can be found in Appendix $\mathrm{H}$.

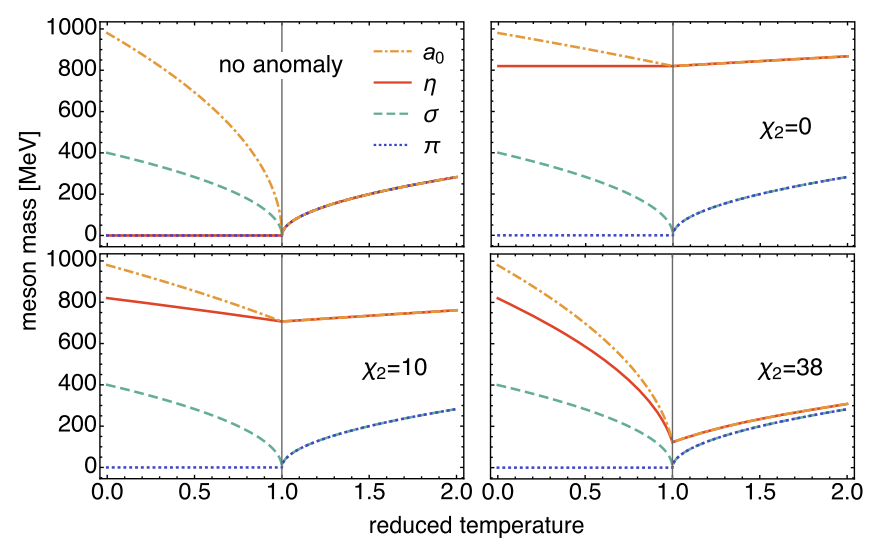

FIG. 1. The masses of mesons as a function of the reduced temperature for two massless quarks in the mean-field approximation. The top left plot shows the spectrum for a $U(1)_{A^{-}}$ symmetric theory. The top right plot is the conventional case where the axial anomaly is induced by instantons of topological charge one. The two figures on the bottom show the effect of additional anomalous symmetry breaking due to instantons of topological charge two for two different values of the corresponding coupling $\chi_{2}$. Unless $\chi_{2}$ is very large, this is negligible.
The splitting between the pion and eta mass is due exclusively to the axial anomaly in the chiral limit. Since $\chi_{2}$ is a quartic coupling, its contribution to the masses is proportional to the chiral condensate. As the condensate melts, this contribution vanishes so that $\chi_{1}$ is the only anomalous contribution to the masses in the symmetric phase. The larger we choose $\chi_{2}$, the smaller $\chi_{1}$ has to be to reproduce the correct vacuum masses. In the chirally symmetric phase $m_{\sigma}=m_{\pi}$ and $m_{\eta}=m_{a_{0}}$, but $m_{\sigma} \neq m_{\eta}$ when $\chi_{1} \neq 0$. Even when $\chi_{1}$ is small, however, we stress that there are still anomalous effects in the chirally symmetric phase from nonzero $\chi_{2}$. These manifest themselves in correlation functions of quartic and higher order.

Needless to say, the effects generated by anomalous coupling from instanton with $Q= \pm 2$ depend upon how large it is in vacuum and how rapidly it decreases with temperature $T$ and quark chemical potential $\mu$. In vacuum, the nature of the dominant fluctuations in topological charge is certainly a formidable problem in nonperturbative physics. While this could be done on the lattice [32-39] or with functional methods [47], to estimate these effects we use a simple gas of dilute instantons. To this end, we adopt a crude bosonization scheme,

$$
2 M^{2} \Phi=\left(\bar{\psi} \psi+\bar{\psi} \gamma \gamma_{5} \psi\right)+\left(\bar{\psi} \vec{\tau} \psi+\bar{\psi} \gamma_{5} \vec{\tau} \psi\right) \vec{\tau}
$$

which yields simple relations between the anomalous meson couplings in Eq. (11), and the corresponding quark couplings in the dilute instanton gas,

$$
\chi_{1}=\frac{\kappa_{1} M^{4}}{2 K_{1,2}}, \quad \chi_{2}=\frac{\kappa_{2} M^{8}}{4 K_{2,2}},
$$

with $\kappa_{1}=\int_{\rho} n_{1}(\rho)\left(8 \pi^{2}\right)^{N_{f}} \rho^{3 N_{f}}$ [4] and $\kappa_{2}$ is given in Eq. (10). The mass scale $M$ is a fundamental parameter of our effective theory. Motivated by the complete computation at one loop order [4], and the partial computation at two loop order [19], for three colors and two massless flavors we take the density of a single instanton in the vacuum to be

$$
n_{1}(\rho)=\frac{d_{\overline{\mathrm{MS}}}}{\rho^{5}}\left(\frac{8 \pi^{2}}{g^{2}}\right)^{6} \exp \left(-\frac{8 \pi^{2}}{g^{2}}\right)
$$

where $g^{2}=g^{2}\left(\rho \Lambda_{\overline{\mathrm{MS}}}\right)$ is the running coupling constant at two loop order and $d_{\overline{\mathrm{MS}}}$ is a renormalization-scheme dependent constant, $\approx 0.00422$ for $N_{c}=3$ and $N_{f}=2$ [48]. The apparent simplicity of our form for the instanton density belies a major assumption that everywhere the coupling $g^{2}$ appears that we can replace it with $g^{2}\left(\rho \Lambda_{\overline{\mathrm{MS}}}\right)$.

This assumption, while admittedly extreme, is both simple and useful. Owing to the interplay between the running coupling from the classical action in the exponential and the factor $\sim g^{-12}$ from the collective coordinate 
Jacobian, $n_{1}(\rho)$ develops a pronounced maximum at $\rho \Lambda_{\overline{\mathrm{MS}}} \approx 1 / 2$. For typical values of $\Lambda_{\overline{\mathrm{MS}}} \approx 300 \mathrm{MeV}$ [49], this implies typical instanton sizes of $\rho \approx 1 / 3 \mathrm{fm}$, which is consistent with the value in an instanton liquid [16-18]. Of course we cannot compute reliably at large $\rho$, since inevitably the instanton size is comparable to the confinement scale, and semiclassical approximations break down.

Since the two anomalous couplings $\chi_{1}$ and $\chi_{2}$ only depend on a single free parameter $M$, we can redo the mean-field analysis of the meson masses and find a unique value for $M$ in the vacuum. From the dilute instanton gas, Eq. (14), with $\Lambda_{\overline{\mathrm{MS}}}=0.3 \mathrm{GeV}$,

$$
\kappa_{1}=3886 \mathrm{GeV}^{-2}, \kappa_{2}=1.819 \times 10^{8} \mathrm{GeV}^{-8} .
$$

In vacuum, this gives $M=0.0953 \mathrm{GeV}$, so from Eq. (13),

$$
\chi_{1}=0.320 \mathrm{GeV}^{2}, \quad \chi_{2}=1.852 .
$$

Of course, $\chi_{1}, \chi_{2}$ and all other anomalous effects are very sensitive to the value chosen for $\Lambda_{\overline{\mathrm{MS}}}$. More to the point, in the confined phase a semiclassical analysis is invalid, and other configurations may contribute [30,31,40,41]. Nevertheless, we expect that our naive computations should give some guide to their overall magnitude.

We conclude by discussing how the dilute instanton gas evaporates as $T$ and $\mu$ increase. For a single instanton we approximate the change to the instanton density for three colors and two flavors as

$n_{1}(\rho, T, \mu)=\exp \left[-\frac{2 \pi^{2}}{g^{2}} \rho^{2} m_{D}^{2}-14 A(\pi \rho T)\right] n_{1}(\rho)$,

where $m_{D}^{2}(T, \mu)$ is the Debye mass at leading order and $A(x)$ has been computed in $[12,13]$. Owing to the screening of the colorelectric field in the medium, the instanton density decreases both with increasing $T$ and $\mu$. We find that instanton effects are decreased to $10 \%$ of their strength in vacuum at about $T \approx 0.7 \Lambda_{\overline{\mathrm{MS}}}$ at $\mu=0$ and $\mu \approx 2.4 \Lambda_{\overline{\mathrm{MS}}}$ at $T=0$. Using realistic values for the critical temperature $T_{c}$ [50] and $\Lambda_{\overline{\mathrm{MS}}}$ [49], we find that instanton effects are significantly suppressed at temperatures $T \gtrsim 1.5 T_{c}$ for $\mu=0$, consistent with lattice results [32-39]. As discussed in Appendix I, at zero temperature in a dilute instanton gas, instantons evaporate only at extremely high densities of $\mu \gtrsim 1.5 \pi T_{c}$. Using $T_{c}=156 \mathrm{MeV}$, this corresponds to baryon chemical potentials of $\mu_{B} \gtrsim 2 \mathrm{GeV}$.

\section{SUMMARY AND OUTLOOK}

We demonstrated that novel effective interactions are generated by instantons of higher topological charge. In general, instantons of topological charge $Q$ give rise to anomalous $2 N_{f}|Q|$-quark interactions. This opens up the possibility to study the effects of the axial anomaly directly for higher correlation function of quarks or hadrons. This includes diquark operators which contribute to color superconductivity [21], and anomalous couplings for tetraquark mesons [28] and heterochiral mesons with spin $J \geq 1$ [29]. The presence of higher topological charge also modifies the $\theta$-dependence and so axion dynamics [51].

\section{ACKNOWLEDGMENTS}

We thank S. Mukherjee for originally asking us about interactions induced by instantons. We also thank M. Creutz, F. Karsch, R. Larsen, P. Petreczky, and E. Shuryak for discussions. R.D.P. is funded by the U.S. Department of Energy under Contract No. DESC0012704; F. R. is supported by the Deutsche Forschungsgemeinschaft (DFG) through Grant No. RE 4174/1-1 and in part by the U.S. Department of Energy under Contract No. DE-SC0012704.

\section{APPENDIX A: CONVENTIONS}

We use a chiral representation for the Euclidean gamma matrices: with the Pauli matrices $\sigma^{i}$,

$$
\sigma^{\mu}=\left(-i \mathbb{1}_{2}, \vec{\sigma}\right)^{\mu}, \quad \bar{\sigma}^{\mu}=\left(i \mathbb{1}_{2}, \vec{\sigma}\right)^{\mu},
$$

then

$$
\gamma^{\mu}=\left(\begin{array}{cc}
0 & i \sigma^{\mu} \\
-i \bar{\sigma}^{\mu} & 0
\end{array}\right)
$$

and

$$
\gamma^{5}=\gamma^{0} \gamma^{1} \gamma^{2} \gamma^{3}=\left(\begin{array}{cc}
-\mathbb{1}_{2} & 0 \\
0 & \mathbb{1}_{2}
\end{array}\right) .
$$

Left- (right-) handed fields have eigenvalue $-1(+1)$ with respect to $\gamma^{5}$. The projection operators on left- and righthanded fields are given by

$$
\mathbb{P}_{L / R}=\frac{\mathbb{1}_{4} \mp \gamma^{5}}{2} .
$$

The matrices in Eq. (A1) can be used to define the basis quaternions,

$$
\bar{\alpha}^{\mu}=i \sigma^{\mu}, \quad \alpha^{\mu}=-i \bar{\sigma}^{\mu} .
$$

We also define

$\sigma^{\mu \nu}=\frac{1}{2}\left(\sigma^{\mu} \bar{\sigma}^{\nu}-\sigma^{\nu} \bar{\sigma}^{\mu}\right), \quad \bar{\sigma}^{\mu \nu}=\frac{1}{2}\left(\bar{\sigma}^{\mu} \sigma^{\nu}-\bar{\sigma}^{\nu} \sigma^{\mu}\right)$,

which are selfdual and anti-selfdual respectively,

$$
\sigma^{\mu \nu}=+\frac{1}{2} \epsilon^{\mu \nu \rho \sigma} \sigma^{\rho \sigma}, \quad \bar{\sigma}^{\mu \nu}=-\frac{1}{2} \epsilon^{\mu \nu \rho \sigma} \bar{\sigma}^{\rho \sigma} .
$$

They are related to the 't Hooft symbols $\eta^{a \mu \nu}$ through the $S U(2)$ color generators $T^{a}$, 


$$
\sigma^{\mu \nu}=-2 \eta^{a \mu \nu} T^{a}, \quad \bar{\sigma}^{\mu \nu}=-2 \bar{\eta}^{a \mu \nu} T^{a} .
$$

We distinguish between Pauli matrices $\sigma^{i}$ for the Dirac matrices, and the $\tau^{a}$ in color space, with $T^{a}=-i \tau^{a} / 2$. For $N_{c}>2$ these generators are given by embedding $S U(2)$ into $S U\left(N_{c}\right)$. The 't Hooft symbols are

$$
\begin{aligned}
& \eta^{a \mu \nu}=\delta^{a \mu} \delta^{\nu 4}-\delta^{a \nu} \delta^{\mu 4}+\epsilon^{a \mu \nu}, \\
& \bar{\eta}^{a \mu \nu}=\delta^{a \nu} \delta^{\mu 4}-\delta^{a \mu} \delta^{\nu 4}+\epsilon^{a \mu \nu},
\end{aligned}
$$

and are also (anti-) selfdual.

\section{APPENDIX B: SMALL INSTANTONS FROM THE ADHM-CONSTRUCTION}

The most general form of an instanton with charge $\pm Q$ is obtained by means of the ADHM construction $[8,10]$. The $Q$-instanton solution is described by a superposition of instantons with unit charge, where each of the constituentinstantons is characterized by a position $z_{i}$, a size $\rho_{i}$, and its orientation in the gauge group, parametrized by a matrix $U_{i}$. We consider the limit in which the distance between the constituent-instantons is large relative to their scale sizes, $\left|z_{i}-z_{j}\right| \gg \rho_{i}$ for all $i \neq j$, which we term small. For a systematic expansion of the ADHM solution for small constituent-instantons see Ref. [10]. Since this is relevant for the construction of the quark zero modes, we outline the construction here.

Without loss of generality, we assume $Q>0$. Antiinstantons can always be obtained trivially be replacing the selfdual matrices $\sigma^{\mu \nu}, \eta^{a \mu \nu}$ by the corresponding antiselfdual matrices $\bar{\sigma}^{\mu \nu}, \bar{\eta}^{a \mu \nu}$; see Appendix A. The most general self-dual gluon field with topological charge $Q$ can be constructed algebraically from a $(Q+1) \times Q$ matrix $M(x)$ whose entries are quaternionic. Each matrix element $M_{a b}$ can therefore be viewed as a $2 \times 2$ matrix,

$$
M_{a b}=\alpha_{\mu} M_{a b}^{\mu},
$$

where the basis quaternions $\alpha$ are defined in Eq. (A5). $M(x)$ is chosen to be linear in spacetime $x$,

$$
M(x)=B-C x,
$$

with the constant quaternionic $(Q+1) \times Q$ matrices $B, C$ and the quaternionic spacetime coordinate $x=\alpha_{\mu} x_{\mu}$. M is required to obey the reality condition,

$$
M^{\dagger}(x) M(x)=R(x)
$$

where $R$ is a real $Q \times Q$ quaternionic matrix. Hence, each entry is proportional to $\alpha_{0}=\mathbb{1}_{2}$. The quaternionic conjugate ${ }^{\dagger}$ is given by

$$
\left(M^{\dagger}\right)_{a b}^{0}=M_{b a}^{0}, \quad\left(M^{\dagger}\right)_{a b}^{i}=-M_{b a}^{i} .
$$

When expressed in terms of Eq. (B1), $M$ can be viewed as a complex $2(Q+1) \times 2 Q$ matrix. We choose the quaternionic representation for convenience. Aside from $M$, the other crucial ingredient is the quaternionic $(Q+1)$ column vector $N(x)$, which obeys

$$
N^{\dagger}(x) M(x)=0, \quad N^{\dagger}(x) N(x)=\mathbb{1}_{2} .
$$

The first equation yields $Q$ equations for the $(Q+1)$ entries of $N$, so $Q$ entries of $N$ can always be expressed in terms of one other entry. The choice of this entry corresponds to a gauge choice for the $Q$-instanton. The second equation is a normalization condition. By solving Eqs. (B3) and (B5) with the ansatz (B2), the $S U(2) Q$-instanton is given by

$$
A_{\mu}^{(Q)}(x)=N^{\dagger}(x) \partial_{\mu} N(x),
$$

where $N$ is determined up to $8 Q-3$ free parameters, corresponding to the complete set of collective coordinates of the instanton for $N_{c}=2$. Only the relative orientation of the constituent-instantons in the gauge group is counted, leaving three parameters for the overall gauge rotation of the solution, so $8 Q$ parameters in all. We follow the explicit construction of $M$ and $N$ in Ref. [10]. The first column of $M$ is given by a vector of $Q$ constant quaternions $q=\left(q_{1}, \ldots, q_{Q}\right)$,

$$
M_{1 j}=q_{j}, \quad j \in[1, \ldots, Q],
$$

and the remaining $Q \times Q$ elements of $M$ are given by

$$
\hat{M}_{i j}(x)=\delta_{i j}\left(z_{i}-x\right)+b_{i j}(x) .
$$

$b_{i j}$ is a quaternionic matrix. The diagonal elements of $\hat{M}$ are parametrized by $z_{i}$, so $b_{i i}=0$. We show below that $z_{i}$ can be interpreted as instanton locations. The reality constraint in Eq. (B3) is fulfilled if $b_{i j}$ is symmetric, $b_{i j}=b_{j i}$, and obeys

$$
\begin{aligned}
& \frac{1}{2}\left(q_{i}^{*} q_{j}-q_{j}^{*} q_{i}\right)+\left(z_{i}-z_{j}\right)^{*} b_{i j} \\
& +\frac{1}{2} \sum_{k=1}^{Q}\left(b_{k i}^{*} b_{k j}-b_{k j}^{*} b_{k i}\right)=R_{i j} .
\end{aligned}
$$

The column vector $N$ is

$$
N(x)=\frac{1}{\sqrt{\xi}}\left(\begin{array}{c}
u \\
-\left[\left(\hat{M}^{\dagger}\right)^{-1} q^{\dagger}\right]_{1} \cdot u \\
\vdots \\
-\left[\left(\hat{M}^{\dagger}\right)^{-1} q^{\dagger}\right]_{Q} \cdot u
\end{array}\right),
$$

where $u$ is an arbitrary, possibly $x$-dependent, unit quaternion. Different $u$ correspond to gauge-equivalent solutions, where $u=\alpha_{0}$ corresponds to singular gauge. $\xi$ is determined from the normalization condition in Eq. (B5), 


$$
\xi=1+q \hat{M}^{-1}\left(\hat{M}^{\dagger}\right)^{-1} q^{\dagger} .
$$

It is left to specify the $b_{i j}$, i.e., to solve Eq. (B9). For this, we restrict ourselves to the limit of small constituentinstantons as this is all we need for our purposes. We first note that every quaternion $q_{i}$ can be represented by a modulus and a phase,

$$
q_{i}=\left|q_{i}\right| U_{i}=\sqrt{q_{i}^{*} q_{i}} U_{i}=\rho_{i} U_{i},
$$

where $U_{i}$ is an $S U(2)$ matrix, and there is no summation over $i$ here. The magnitude of $q_{i}$ can be interpreted as the scale of the instanton, $\rho_{i}=\left|q_{i}\right| . U_{i}$ parametrizes the orientation in the gauge group. In the limit of small constituent-instantons, we introduce a small parameter $\zeta$ and replace

$$
q_{i} \rightarrow \zeta q_{i},
$$

where $q_{i}$ is kept fixed. We then consider the instanton scale to be small relative to the instanton separations, $\zeta\left|q_{i}\right| \ll\left|z_{i}-z_{j}\right|$, and expand Eq. (B9) to leading order in $\zeta$. The solution is unchanged if $\hat{M} \rightarrow T \hat{M}$, where $T$ is an orthogonal quaternionic matrix. Choosing

$$
T_{i j}=\delta_{i j}+\frac{R_{i j}}{\left(z_{i}-z_{j}\right)^{2}}+\mathcal{O}\left(\zeta^{4}\right),
$$

cancels the right-hand side of Eq. (B9). To this order, then, one finds

$$
b_{i j}=\frac{1}{2} \frac{z_{i}-z_{j}}{\left(z_{i}-z_{j}\right)^{2}}\left(q_{i}^{*} q_{j}-q_{j}^{*} q_{i}\right) .
$$

Thus, for small constituent-instantons we can neglect $b_{i j}$ and $M$ becomes

$M(x)=\left(\begin{array}{ccc}q_{1} & \cdots & q_{Q} \\ \left(z_{1}-x\right) & \cdots & 0 \\ \vdots & \ddots & \vdots \\ 0 & \cdots & \left(z_{Q}-x\right)\end{array}\right)+\mathcal{O}\left(\zeta^{2}\right)$.

The dominant contribution to the determinant of zero modes for the $Q$-instanton comes from distances large relative to the size of each constituent-instanton $\left|x-z_{i}\right| \gg$ $\rho_{i}$. The constant quaternionic matrices $B$ and $C$ in Eq. (B2) are then

$B=\left(\begin{array}{ccc}q_{1} & \cdots & q_{Q} \\ z_{1} & \cdots & 0 \\ \vdots & \ddots & \vdots \\ 0 & \cdots & z_{Q}\end{array}\right), \quad C=\left(\begin{array}{ccc}0 & \cdots & 0 \\ \mathbb{1}_{2} & \cdots & 0 \\ \vdots & \ddots & \vdots \\ 0 & \cdots & \mathbb{1}_{2}\end{array}\right)$.

With this, $\xi=\xi_{0}+\mathcal{O}\left(\xi^{2}\right)$ in Eq. (B11), where

$$
\xi_{0}(x)=1+\sum_{i=1}^{Q} \frac{\rho_{i}^{2}}{\left(x-z_{i}\right)^{2}},
$$

and $N(x)$ becomes

$$
N(x)=\frac{1}{\sqrt{\xi_{0}}}\left(\begin{array}{c}
u \\
\frac{x-z_{1}}{\left(x-z_{1}\right)^{2}} q_{1}^{*} \cdot u \\
\vdots \\
\frac{x-z_{Q}}{\left(x-z_{Q}\right)^{2}} q_{Q}^{*} \cdot u
\end{array}\right)+\mathcal{O}\left(\zeta^{2}\right) .
$$

Choosing $u=\alpha_{0}$ and plugging this into Eq. (B6) then yields the small $Q$-instanton,

$$
A_{\mu}^{(Q)}(x)=\frac{1}{\xi_{0}(x)} \sum_{i=1}^{Q} U_{i} \bar{\sigma}^{\mu \nu} U_{i}^{\dagger} \rho_{i}^{2} \frac{\left(x-z_{i}\right)_{\nu}}{\left|x-z_{i}\right|^{4}},
$$

with $\bar{\sigma}^{\mu \nu}$ defined in Eq. (A6). If all constituent-instantons are aligned in color space, $U_{i}=U_{j}$ for all $i, j=1, \ldots, Q$, this reduces to 't Hooft's solution [7].

A key feature of the limit of small constituent-instantons is that the field of the $Q$-instanton, Eq. (B20), is only significant when $x$ close to the location of one of the constituent-instantons, at $z_{i}$. In the vicinity of each $z_{i}$, Eq. (B20) looks like a $Q=1$ instanton in singular gauge,

$\left.A_{\mu}^{(Q)}(x)\right|_{\left|x-z_{i}\right| \approx \mathcal{O}\left(\rho_{i}\right)}=U_{i} \bar{\sigma}^{\mu \nu} U_{i}^{\dagger} \frac{\rho_{i}^{2}}{\left(x-z_{i}\right)^{2}} \frac{\left(x-z_{i}\right)_{\nu}}{\left(x-z_{i}\right)^{2}+\rho_{i}^{2}}$.

To leading order in powers of $\rho_{i}^{2} /\left(z_{i}-z_{j}\right)^{2}$, in the saddle point approximation the generating functional for a small $Q$-instanton factorizes into a product of generating functionals in the backgrounds of single instantons, Eq. (2). See Refs. [11,20] for a more detailed discussion of this factorization.

\section{APPENDIX C: QUARK ZERO MODES FOR A SMALL $Q$-INSTANTON}

In the presence of a $Q$-instanton quarks have zero modes,

$$
D^{(Q)} \psi_{f i}^{(Q)}=0,
$$

where $D^{(Q)}=\gamma_{\mu}\left(\partial_{\mu}+A_{\mu}^{(Q)}\right)$ is the Dirac operator in the fundamental representation; remember that $f$ is an index for flavor. The Atiyah-Singer index theorem demonstrates that gauge field configurations with topological charge $Q$ produce $N_{f}|Q|$ left-handed (for $Q>0$ ) or right-handed (for $Q<0$ ) quark zero modes [6,52]. With the ADHM construction the zero modes are [11]

$$
\psi_{f i}^{(Q)}=\nu\left(N^{\dagger} C R^{-1}\right)_{i} \cdot \varphi ;
$$

this is a left-handed Weyl spinor, $\varphi=\epsilon / \sqrt{2}$, and $\nu$ is a normalization constant. For small constituent-instantons with $Q=2$ we use Eqs. (B16), (B17) and (B19) to find 


$$
\begin{aligned}
C & =\left(\begin{array}{cc}
0 & 0 \\
\mathbb{1}_{2} & 0 \\
0 & \mathbb{1}_{2}
\end{array}\right), \\
R & =\left(\begin{array}{cc}
\left(x-z_{1}\right)^{2}+\rho_{1}^{2} & q_{1}^{*} q_{2} \\
q_{2}^{*} q_{1} & \left(x-z_{2}\right)^{2}+\rho_{2}^{2}
\end{array}\right) .
\end{aligned}
$$$$
R^{-1}=\frac{1}{\operatorname{det} R}\left(\begin{array}{cc}
R_{22} & -R_{12} \\
-R_{21} & R_{11}
\end{array}\right)
$$

with

Note that to leading order $R$ is diagonal, $\operatorname{diag}\left[\left(x-z_{1}\right)^{2}\right.$, $\left.\left(x-z_{2}\right)^{2}\right]$; the other terms are $\mathcal{O}\left(\zeta^{2}\right)$. Since $R$ is real, we use

$$
\operatorname{det} R=\left(x-z_{1}\right)^{2}\left(x-z_{2}\right)^{2}+\rho_{2}^{2}\left(x-z_{1}\right)^{2}+\rho_{1}^{2}\left(x-z_{2}\right)^{2} .
$$

This yields

$$
\begin{aligned}
\psi_{f 1}^{(2)}(x)= & \nu \frac{U_{1} \rho_{1}}{\left|x-z_{1}\right|} \gamma_{\mu}\left[\left(x-z_{1}\right)_{\mu}\left(\left(x-z_{2}\right)^{2}+\rho_{2}^{2}\right)-\rho_{2}^{2}\left(x-z_{2}\right)_{\mu} \frac{\left(x-z_{1}\right)^{2}}{\left(x-z_{2}\right)^{2}}\right] \\
& \times \frac{\left|x-z_{2}\right|}{\left[\left(x-z_{1}\right)^{2}\left(x-z_{2}\right)^{2}+\rho_{2}^{2}\left(x-z_{1}\right)^{2}+\rho_{1}^{2}\left(x-z_{2}\right)^{2}\right]^{3 / 2}} \varphi_{R}, \\
\psi_{f 2}^{(2)}(x)= & \left.\psi_{f 1}^{(2)}(x)\right|_{1 \leftrightarrow 2},
\end{aligned}
$$

with the right-handed spinor,

$$
\varphi_{R}^{\alpha c}=\frac{1}{\sqrt{2}}\left(\begin{array}{l}
0 \\
\epsilon
\end{array}\right)_{\alpha c},
$$

where $\alpha$ is a spinor index, $c$ is a $S U(2)$ color index and $\epsilon$ is the antisymmetric tensor. Because of the factor of $\gamma_{\mu}$ in Eq. (C6), the zero mode $\psi_{f i}^{(Q)}(x)$ is left-handed when $Q>0$, as required by the index theorem. For an antiinstanton, $Q<0$, one simply has to replace $\varphi_{R}$ by

$$
\varphi_{L}^{\alpha c}=\frac{1}{\sqrt{2}}\left(\begin{array}{l}
\epsilon \\
0
\end{array}\right)_{\alpha c} .
$$

To turn the Weyl into Dirac spinors we use $\gamma_{\mu} \varphi_{R}=\bar{\alpha}_{\mu} \varphi$, and Eq. (B12) to express the quaternions $q_{i}$ in terms of the sizes and gauge group orientations of the constituentinstantons. As a results, the $i$ th quark zero mode carries the gauge group orientation of the corresponding constituent-instanton. If they had the same orientation, $U_{1}=U_{2}$, they would be identical to the zero modes that follow from 't Hooft's solution for the aligned instanton [11].

For small constituent-instantons,

$$
\left|R_{12}\right| \equiv\left|z_{1}-z_{2}\right| \gg \rho_{1}, \rho_{2}
$$

We consider the behavior of the zero modes far from the constituent-instantons,

$$
\left|x-z_{1}\right|,\left|x-z_{2}\right| \gg \rho_{1}, \rho_{2} .
$$

Since the $Q$-instanton is an extended object, in general it generates a quark interaction which is nonlocal. We wish to extract the local term, in which the sizes of the constituent-instantons can be neglected. In this limit, Eq. (C6) is approximately,

$$
\begin{aligned}
\psi_{f 1}^{(2)}(x) \approx & \nu \frac{U_{1} \rho_{1}}{\left[\left(x-z_{1}\right)^{2}+\rho_{1}^{2}\right]^{3 / 2}} \frac{\gamma_{\mu}\left(x-z_{1}\right)_{\mu}}{\left|x-z_{1}\right|} \varphi_{R} \\
& -\nu \frac{U_{1} \rho_{2}}{\left[\left(x-z_{2}\right)^{2}+\rho_{2}^{2}\right]^{3 / 2}} \frac{\gamma_{\mu}\left(x-z_{2}\right)_{\mu}}{\left|x-z_{2}\right|} \\
& \times \frac{\rho_{1} \rho_{2}\left|x-z_{1}\right|}{\left[\left(x-z_{1}\right)^{2}+\rho_{1}^{2}\right]^{3 / 2}} \varphi_{R} \\
= & \psi_{f 1}\left(x, z_{1}\right)-\mathbb{X}_{1}\left(x, z_{1}\right) \hat{\psi}_{f 2}\left(x, z_{2}\right),
\end{aligned}
$$

and analogously for the second zero mode $\psi_{f 2}^{(2)}(x)$. In this approximation, we drop a term $\rho_{1}^{2} \rho_{2}^{2}$ in the denominator as subleading and then used Eq. (C10) to reduce the expression. We define the $Q=1$ zero modes as

$\psi_{f i}\left(x, z_{i}\right)=\nu \frac{U_{i} \rho_{i}}{\left[\left(x-z_{i}\right)^{2}+\rho_{i}^{2}\right]^{3 / 2}} \frac{\gamma_{\mu}\left(x-z_{i}\right)_{\mu}}{\left|x-z_{i}\right|} \varphi_{R}$,

$\hat{\psi}_{f i}\left(x, z_{i}\right)=U_{j} U_{i}^{\dagger} \psi_{f i}\left(x, z_{i}\right)$,

and

$$
\mathbb{X}_{i}\left(x, z_{i}\right)=\frac{\rho_{1} \rho_{2}\left|x-z_{i}\right|}{\left[\left(x-z_{i}\right)^{2}+\rho_{i}^{2}\right]^{3 / 2}}
$$

The first zero mode for $Q=2$ is a sum of a zero mode concentrated at $z_{1}$ plus an overlap term, which is $\mathbb{X}_{i}$ times the zero mode concentrated at $z_{2}$; that for the second zero mode is similar, with the exchange of $1 \leftrightarrow 2$. The overlap term is $\mathcal{O}\left(\zeta^{3}\right)$. To leading order, i.e., $\mathcal{O}\left(\zeta^{1}\right)$, the zero modes reduce to the $Q=1$ zero modes, $\psi_{f 1}^{(2)}=\psi_{f 1}+\mathcal{O}\left(\zeta^{3}\right)$. 


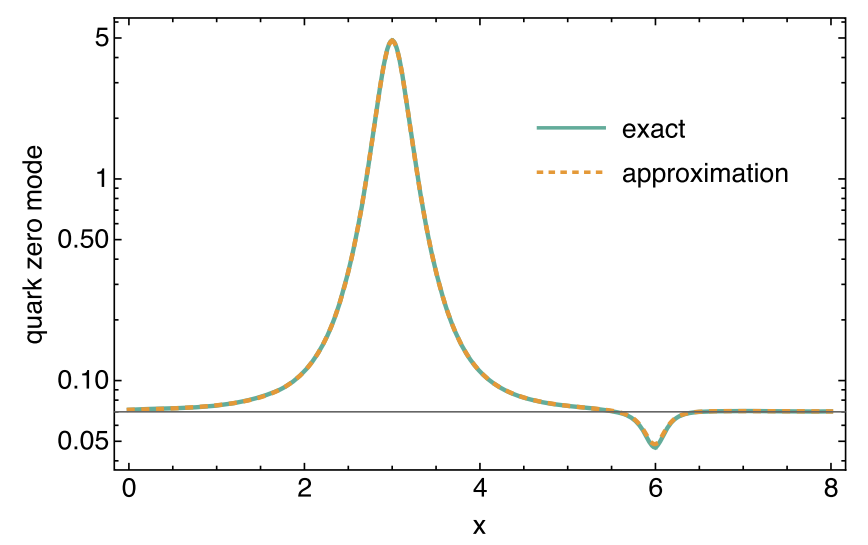

FIG. 2. Comparison between the exact form of the $Q=2$ quark zero mode $\psi_{f 1}^{(2)}(x)$ for small constituent-instantons and our approximation in Eq. (C11). We used the parameters $z_{1}=3, z_{2}=$ 6 and $\rho_{1}=\rho_{2}=0.21$ for this plot. An offset of +0.07 was added so that a logarithmic scale could be used along the $y$-axis, since the baseline of the zero mode vanishes. The scalar function plotted is defined in Eq. (C14).

In Fig. 2 we compare the exact quark zero mode in the limit of small constituent-instantons, Eq. (C6), to the approximate form in Eq. (C11). For this figure, we project onto the scalar part of the zero mode $\psi_{f 1}^{(2)}$ via

$$
\varphi_{R}^{\dagger} \frac{\gamma_{\mu}\left(x-z_{1}\right)_{\mu}}{\left|x-z_{1}\right|} \psi_{f 1}^{(2)}(x),
$$

for the configuration $\left(x-z_{1}\right) \cdot\left(x-z_{2}\right)=\left|x-z_{1}\right|\left|x-z_{2}\right|$, so that the function depends only upon the relative distances. Our approximate form is very good even close to $z_{1}$ and $z_{2}$ for $\rho /|R| \lesssim 0.3$. At leading order the normalization constant $\nu$ is determined via

$$
\int d^{4} x \psi_{f i}^{(2) \dagger}(x) \psi_{f i}^{(2)}(x) \approx \int d^{4} x \psi_{f i}^{\dagger}\left(x, z_{i}\right) \psi_{f i}\left(x, z_{i}\right) \equiv 1,
$$

which gives $\nu=\sqrt{2} / \pi$.

To make the computations more transparent we use a graphical representation of the zero modes in Eq. (C11),

$$
\begin{aligned}
& \psi_{f 1}^{(2)}(x)=(\underbrace{}_{f 1})_{f}()_{f 1}-\mathbb{X}_{1}\left(\bigwedge_{f 2},\right. \\
& \psi_{f 2}^{(2)}(x)=(\neg)_{f 2}=(\preceq)_{f 2}-\mathbb{X}_{2}(\bigwedge)_{f 1},
\end{aligned}
$$

where the left peak is located at $z_{1}$ and the right peak at $z_{2}$.

When the constituent-instantons are small, Eq. (C10), the zero mode in Eq. (C12) becomes

$$
\psi_{f i}\left(x, z_{i}\right) \approx \nu \frac{U_{i} \rho_{i} \gamma_{\mu}\left(x-z_{i}\right)_{\mu}}{\left|x-z_{i}\right|^{4}} \varphi_{R}=2 \pi^{2} \nu U_{i} \rho_{i} \Delta\left(x-z_{i}\right) \varphi_{R},
$$

where $\Delta(x-z)$ is the free propagator of a massless quark,

$$
\Delta(x-z)=\frac{\gamma_{\mu}(x-z)_{\mu}}{2 \pi^{2}|x-z|^{4}} .
$$

Hence, for $Q=2$ the quark zero modes reduce to a sum of free quark propagators and the overlap term,

$\psi_{f 1}^{(2)}(x) \propto U_{1}\left[\rho_{1} \Delta\left(x-z_{1}\right)-\rho_{2} \mathbb{X}_{1}\left(x, z_{1}\right) \Delta\left(x-z_{2}\right)\right] \varphi_{R}$.

This expression is essential in extracting the effective Lagrangian of quarks from the product of zero modes below.

Our results can be generalized to higher topological charge. It is less obvious how to move away from the limit of small constituent-instantons, since then the terms generated in an effective Lagrangian involve derivatives of the quark fields, and so are of higher order in a derivative expansion.

\section{APPENDIX D: GENERATING FUNCTIONAL FOR A SMALL $Q$-INSTANTON TO LEADING ORDER}

We begin with the generating functional in a $Q$-instanton background, focusing on the determinant of quark zero modes to leading order in the limit of small constituentinstantons. We evaluate the generating functional in the saddle point approximation to leading order, where the stationary point is given by an instanton of topological charge $Q$. This is natural, as (anti)self-dual, topological gauge field configurations are minima of the classical Yang-Mills action, assuming only that the action is finite [2]. The generating functional is

$$
Z^{(Q)}[J]=\int \mathcal{D} \chi \exp \left\{-S\left[\chi+\chi^{(Q)}\right]+\int d^{4} x \bar{\psi}(x) J(x) \psi(x)\right\},
$$

where $\chi=\left(A_{\mu}, c, \bar{c}, \psi, \bar{\psi}\right)$ is the fluctuating field of gluons, ghosts and quarks, $S[\chi]$ is the gauge-fixed action in Euclidean spacetime, $\chi^{(Q)}=\left(A_{\mu}^{(Q)}, 0,0,0,0\right)$ is the $Q$ instanton background field, and $J$ a source for quarks. To leading order in the saddle point approximation, the action $S\left[\chi+\chi^{(Q)}\right]$ is expanded in the $\chi^{(Q)}$. The linear terms vanish by the equations of motion, and the $\chi$ are integrated to quadratic order. In the presence of the $Q$ instanton, all fields have zero modes related to the invariance of the action under translations, dilatations and global gauge rotations. There are $4 N_{c}|Q|$ collective 
coordinates describing their position $\left(z_{i}\right)$, size $\left(\rho_{i}\right)$, and orientation in the gauge group $\left(U_{i}\right)$. The set of these coordinates is denoted by $C_{Q}$. Fluctuations in the directions of zero modes are large, so while the nonzero modes can be computed in the Gaussian approximation, the zero modes have to be treated exactly. To this end, one changes the integration over zero modes to an integration over collective coordinates, giving rise to a Jacobian $\mathcal{J}$. This yields the $Q$-instanton density,

$$
\begin{aligned}
n_{Q}\left(C_{Q}\right) \equiv & \mathrm{e}^{-8 \pi^{2}|Q| / g^{2}}\left(\operatorname{det}_{\varnothing} \mathcal{M}_{A}\right)^{-1 / 2} \\
& \times\left(\operatorname{det}_{\varnothing} \mathcal{M}_{c}\right)\left(\operatorname{det}_{\varnothing} \not\right)(\operatorname{det} \mathcal{J}) ;
\end{aligned}
$$

$\mathcal{M}_{A}, \mathcal{M}_{c}$, and $\mathcal{M}_{\psi}=D^{(Q)}+J$ are the inverse propagators of the gluons, ghosts, and quarks, respectively. det $_{\varnothing}$ denotes the determinant over nonzero modes. To renormalize the contributions of large eigenvalues, it is understood that all nonzero-mode determinants are normalized with the determinant at vanishing gluon background field. In this semiclassical approximation, the generating functional becomes

$$
Z^{(Q)}[J]=\int d C_{Q} n_{Q}\left(C_{Q}\right) \operatorname{det}_{0}(J) .
$$

$\operatorname{det}_{0}(J)$ is the determinant of the source $J$ in the space of quark zero modes. This was first computed by 't Hooft in Ref. [4] for $Q=1$ and $N_{c}=2$. The generalization to $N_{c} \geq 3$ is given in Ref. [43]; for $|Q|>1$, solutions are only known in certain limits, see e.g., Refs. [11,20].

For topological charge $Q$ at leading order in the limit of small constituent-instantons, the gauge field is that of Eq. (B20). The off diagonal term in the quaternionic matrix $R$ in Eq. (C3) can be neglected, and one can set $\xi_{0}=1$ in Eq. (B18). The quark zero modes in Eq. (C6) then reduce to the $Q=1$ ones in Eq. (C12), and the zero mode determinant becomes

$$
\begin{aligned}
\operatorname{det}_{0}(J) & =\operatorname{det} \int d^{4} x_{f i} \psi_{f i}^{(Q) \dagger}\left(x_{f i}\right) J_{i j}^{f g}\left(x_{f i}\right) \psi_{g j}^{(Q)}\left(x_{f i}\right) \\
& \approx \operatorname{det} \int d^{4} x_{f i} \psi_{f i}^{\dagger}\left(x_{f i}, z_{i}\right) J_{i j}^{f g}\left(x_{f i}\right) \psi_{g j}\left(x_{f i}, z_{i}\right),
\end{aligned}
$$

where we do not sum over the indices. The quark source $J$ is a $\left(N_{f} Q \times N_{f} Q\right)$-matrix in the space of zero modes, but it suffices to consider a diagonal $J, J_{i i}^{f f} \equiv J_{f i}$. We will match the zero mode determinant to an effective multiquark interaction, so the different contributions to the determinant can be obtained by permutation of the quark fields [cf. Eq. (G2)]. Using the explicit form of the quark zero modes in Eq. (C12), the diagonal elements yield

$$
\left.\operatorname{det}_{0}(J)\right|_{\text {diagonal }}=\prod_{i=1}^{Q} \rho_{i}^{3 N_{f}} \prod_{f=1}^{N_{f}} \int d^{4} x_{f i} \mathcal{F}_{i}\left(x_{f i} ; z_{i}, \rho_{i}, U_{i}\right),
$$

with the zero mode correlation function,

$$
\mathcal{F}_{i}(x ; z, \rho, U)=\frac{2}{\pi^{2}} \frac{U^{\dagger} \varphi_{R}^{\dagger} \gamma_{\mu}(x-z)_{\mu} \bar{J}_{i}(x) \gamma_{\nu}(x-z)_{\nu} \varphi_{R} U}{(x-z)^{2}\left[(x-z)^{2}+\rho^{2}\right]^{3}} .
$$

The quark zero modes have mass dimension two, while that of the source $J$ is zero. We then introduce a source with canonical mass dimension,

$$
\bar{J}_{f i}=\rho_{i}^{-1} J_{f i}
$$

When $\left|x_{f i}-z_{i}\right| \gg \rho_{i}$, we can relate the zero modes to free quark propagators, Eq. (C17), and the quark zero mode correlations are related to those of free quarks,

$$
\mathcal{F}_{i}(x ; z, \rho, U) \approx 8 \pi^{2} U^{\dagger} \varphi_{R}^{\dagger} \Delta(x-z) \bar{J}_{i}(x) \Delta(x-z) \varphi_{R} U .
$$

Note that this is independent of the instanton scale $\rho$. Since the generating functional involves an integral over the collective coordinates, we can express Eq. (D5) as

$$
\begin{aligned}
\left.\operatorname{det}_{0}(J)\right|_{\text {diagonal }} & =\left[\rho_{1}^{3 N_{f}} \int d^{4} x_{f 1} \mathcal{F}_{i}\left(x_{f 1} ; z_{1}, \rho_{1}, U_{1}\right)\right]^{Q} \\
& =\left[\left.\operatorname{det}_{0}(J)\right|_{Q=1}\right]^{Q} .
\end{aligned}
$$

Thus to leading order for small constituent-instantons, the determinant over quark zero modes factorizes into a product of contributions from terms with $Q=1$.

As discussed in Refs. [10,11,20], the functional determinants of the gluons and ghosts factorize to order $\sim \rho^{4} /|R|^{4}$ for small constituent-instantons. Hence, the generating functional factorizes,

$$
\begin{aligned}
Z^{(Q)} & {[J] \underset{\operatorname{small}}{\rightarrow} \frac{1}{Q !}\left[\left.\int d C_{1} n_{1}\left(\rho_{1}\right) \operatorname{det}_{0}(J)\right|_{Q=1}\right]^{Q} } \\
= & \frac{1}{Q !}\left(Z^{(1)}[J]\right)^{Q},
\end{aligned}
$$

where $Z^{(1)}[J]$ is the result for $Q=1$. If $\mathcal{V}$ is the volume of space-time, this is $\sim \mathcal{V}^{|Q|}$, this is evidently the expansion of the exponential of the term with $|Q|=1$. Instead, what we need is a subleading term, proportional to a single power of $\mathcal{V}$. We compute this term explicitly for $Q=2$ in the next sections. 


\section{APPENDIX E: GENERATING FUNCTIONAL FOR $Q=2$ AND ONE FLAVOR}

Here we compute the generating functional beyond leading order in the limit of small constituent-instantons for $Q=2$. The determinant over the quark zero modes follow from Eq. (C11). We begin with the case of a single flavor and so drop the flavor index to obtain

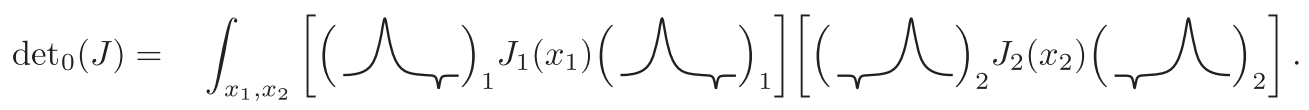

We adopt a shorthand notation where $\int_{x_{1}}=\int d^{4} x_{1}$, etc. For small constituent-instantons, Eqs. (C11) and (C16) show that this contains various contributions. The integrations over the locations of the sources at $x_{1}$ and $x_{2}$ is always present, and so we suppress it. Instead we concentrate on the integrals over the locations of the instantons, at $z_{1}$ and $z_{2}$. Taking the dominant piece from each zero mode,

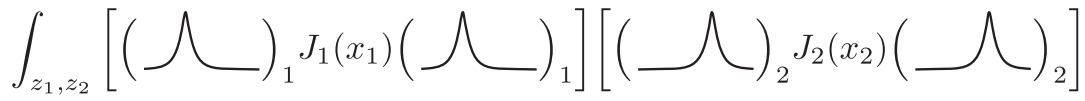

$$
\begin{aligned}
& =\int_{z_{1}}\left\{\left[(\Lambda)_{1} J_{1}\left(x_{1}\right)(\Lambda)_{1}\right]\right\} \int_{z_{2}}\left\{\left[(\Omega)_{2} J_{2}\left(x_{2}\right)(\Omega)_{2}\right]\right\} \text {. }
\end{aligned}
$$

This term has no overlap between the contributions at $z_{1}$ and $z_{2}$, and so completely decomposes into two contributions from independent instantons with $|Q|=1$. These are $\sim \mathcal{V}^{2}$, as discussed in the previous section.

We need to extract local terms $\sim \mathcal{V}$, which are given by

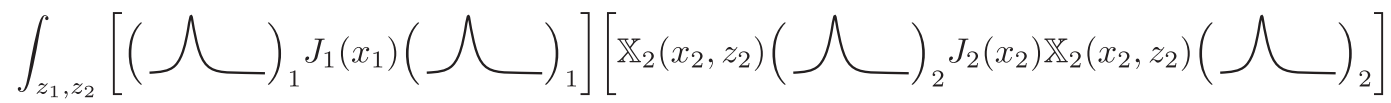

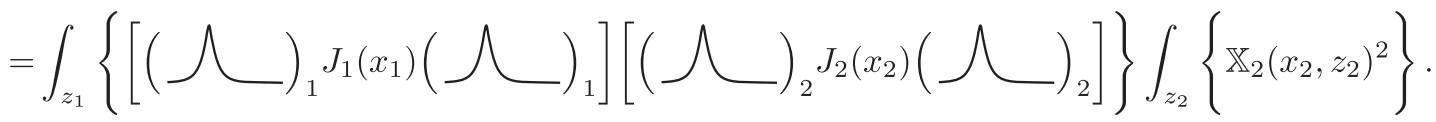

and

$$
\begin{aligned}
& \int_{z_{1}, z_{2}}\left[\mathbb{X}_{1}\left(x_{1}, z_{1}\right)(\preceq)_{1} J_{1}\left(x_{1}\right) \mathbb{X}_{1}\left(x_{1}, z_{1}\right)(\preceq)_{1}\right]\left[(\preceq)_{2} J_{2}\left(x_{2}\right)(\preceq)_{2}\right] \\
& =\int_{z_{1}}\left\{\mathbb{X}_{1}\left(x_{1}, z_{1}\right)^{2}\right\} \int_{z_{2}}\left\{\left[(\preceq)_{1} J_{1}\left(x_{1}\right)(\Omega)_{1}\right]\left[(\Omega)_{2} J_{2}\left(x_{2}\right)(\Omega)_{2}\right]\right\} \text {. }
\end{aligned}
$$

These terms are given by four $Q=1$ zero modes centered around a single $z_{i}$. The integral

$$
\mathcal{I}_{1, i}=\int_{z_{i}} \mathbb{X}_{i}\left(x_{i}, z_{i}\right)^{2}
$$

represents the "leakage" from the constituent-instanton at $z_{2}$ to $z_{1}$, or vice versa. This integral can be done analytically,

$$
\mathcal{I}_{1, i}\left(\rho_{1}, \rho_{2}\right)=\rho_{1}^{2} \rho_{2}^{2} \int d^{4} z_{i} \frac{\left(x_{i}-z_{i}\right)^{2}}{\left[\left(x_{i}-z_{i}\right)^{2}+\rho_{i}^{2}\right]^{3}}=\pi^{2} \rho_{1}^{2} \rho_{2}^{2}\left[\ln \left(\frac{R_{0}^{2}}{R_{0}^{2}+\rho_{i}^{2}}\right)-\frac{R_{0}^{2}\left(3 R_{0}^{2}+2 \rho_{i}^{2}\right)}{2\left(R_{0}^{2}+\rho_{i}^{2}\right)^{2}}\right] .
$$

For a single flavor this overlap integral is dominated by large distances, $\left|x-z_{i}\right|$. There is a logarithmic divergence in the infrared, which we cut off at a scale $R_{0} \gg \rho_{i}$. Presumably $R_{0}$ is related to the average separation between instantons and anti-instantons in the vacuum [16-18]. 
With this, Eq. (E3) becomes

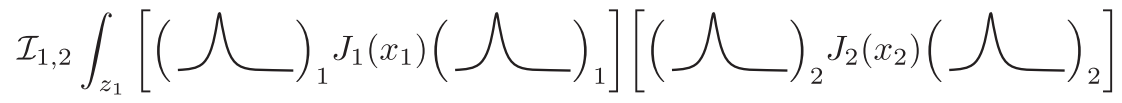

$$
\begin{aligned}
& =\mathcal{I}_{1,2} \int_{z_{1}}\left[\psi_{11}^{\dagger}\left(x_{1}, z_{1}\right) J_{1}\left(x_{1}\right) \psi_{11}\left(x_{1}, z_{1}\right)\right]\left[\widehat{\psi}_{11}^{\dagger}\left(x_{2}, z_{1}\right) J_{2}\left(x_{2}\right) \widehat{\psi}_{11}\left(x_{2}, z_{1}\right)\right] \\
& =\mathcal{I}_{1,2} \int_{z_{1}}\left[\rho_{1}^{3} \mathcal{F}_{1}\left(x_{1} ; z_{1}, \rho_{1}, U_{1}\right)\right]\left[\rho_{1}^{2} \rho_{2} \mathcal{F}_{2}\left(x_{2} ; z_{1}, \rho_{1}, U_{2}\right)\right] \text {, }
\end{aligned}
$$

where we used Eqs. (C11), (D6) and (D7). For Eq. (E4) we find the same result, but with the charge indices ${ }_{1}$ and 2 interchanged. The peculiar powers of the instanton sizes in Eq. (E7), as compared to the leading-order result in Eq. (D9), arise from the overlap of the zero modes. The contributions of the first zero mode, $\psi_{11}^{(2)}$, and the second zero mode, $\psi_{12}^{(2)}$, centered around the same point $z_{1}$, each have the scale $\rho_{1}$ so that they each contribute $\rho_{1}^{2}$ to the determinant. Rescaling the two quark sources $J_{1}$ and $J_{2}$ according to Eq. (D7) yields additional factors $\rho_{1}$ and $\rho_{2}$ respectively. For a single quark flavor, the local quark zero mode determinant therefore goes like $\rho_{1}^{5} \rho_{2}$, instead of $\rho_{1}^{3} \rho_{2}^{3}$ for the nonlocal contribution at leading order.

We have shown that the overlap between the different quark zero modes arises only beyond leading order in the limit of small constituent instantons. This is essential for deriving a local effective action $\sim \mathcal{V}$. Remarkably, even to order $\zeta^{3}$, the gauge contribution to the path integral factorizes and we can still use Eq. (B20) for the 2-instanton [10]. So only corrections of $\mathcal{O}\left(\zeta^{3}\right)$ for the quark zero mode determinant need to be included. With the results above, the local part of the $Q=2$ partition function $Z^{(2)}[J]$, Eq. (2), for $N_{f}=1$ reduces to

$$
\left.Z^{(2)}[J]\right|_{\text {local }}=\frac{1}{2} \int d \rho_{1} n_{1}\left(\rho_{1}\right) \rho_{1}^{5} \int d \rho_{2} n_{1}\left(\rho_{2}\right) \rho_{2} \mathcal{I}_{1,2} \int d^{4} z_{1} \prod_{i=1}^{2}\left\{\int d U_{i} \int d^{4} x_{i} \mathcal{F}_{i}\left(x_{i} ; z_{1}, U_{i}\right)\right\}+(1 \leftrightarrow 2) .
$$

Since this expression is symmetric under the exchange of the topological charge indices $1_{1}$ and ${ }_{2}$, we finally arrive at

$$
\left.Z^{(2)}[J]\right|_{\text {local }}=\int d \rho_{1} n_{1}\left(\rho_{1}\right) \rho_{1}^{5} \int d \rho_{2} n_{1}\left(\rho_{2}\right) \rho_{2} \mathcal{I}_{1,2} \int d^{4} z_{1} \prod_{i=1}^{2}\left\{\int d U_{i} \int d^{4} x_{i} \mathcal{F}_{i}\left(x_{i} ; z_{1}, U_{i}\right)\right\},
$$

where the $Q=1$ instanton density $n_{1}$ is given by Eq. (14).

\section{APPENDIX F: GENERATING FUNCTIONAL FOR $Q=2$ AND $N_{f} \geq 2$}

The discussion for two or more flavors is a straightforward generalization of that for a single flavor. Taking the quark source to be diagonal, the determinant is

$$
\left.\operatorname{det}_{0}(J)\right|_{\text {diag }}=\prod_{f=1}^{N_{f}}\left\{\int _ { x _ { f 1 } } \left[(\underbrace{J_{f 1}\left(x_{f 1}\right)}_{f 1}(\underbrace{\gamma_{f 1}}_{f 1}] \int_{x_{f 2}}\left[\left(\boldsymbol{\gamma}_{f_{2}} J_{f 2}\left(x_{f 2}\right)\left(\boldsymbol{\gamma}_{f 2}\right]\right\} .\right.\right.\right.
$$

There are numerous contributions $\sim \mathcal{V}^{2}$. Assuming that the instantons are uncorrelated, the leading term is

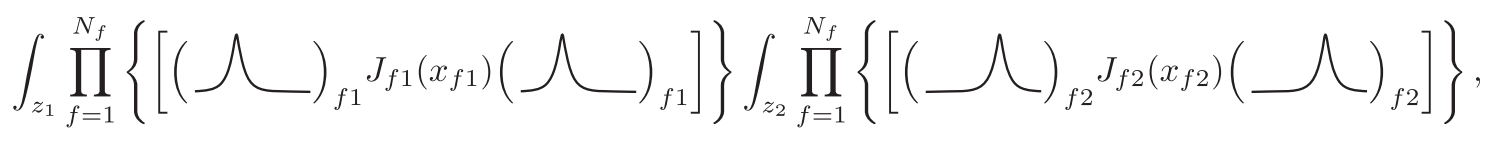

as discussed in Appendix D. 
The terms of interest, $\sim \mathcal{V}$, are given by

$$
\int_{z_{1}} \prod_{f=1}^{N_{f}}\left\{\left[\left(\mathcal { U } _ { f 1 } J _ { f 1 } ( x _ { f 1 } ) ( \underbrace { } _ { f 1 } ] \left[(\underbrace{}_{f 2} J_{f 2}\left(x_{f 2}\right)(\underbrace{}_{f 2}]\} \int_{z_{2}} \prod_{f=1}^{N_{f}}\left\{\mathbb{X}_{2}\left(x_{f 2}, z_{2}\right)^{2}\right\}\right.\right.\right.\right.
$$

and

$$
\int_{z_{1}} \prod_{f=1}^{N_{f}}\left\{\mathbb{X}_{1}\left(x_{f 1}, z_{1}\right)^{2}\right\} \int_{z_{2}} \prod_{f=1}^{N_{f}}\left\{\left[(\bigwedge)_{f 1} J_{f 1}\left(x_{f 1}\right)(\preceq)_{f 1}\right]\left[(\preceq)_{f 2} J_{f 2}\left(x_{f 2}\right)(\preceq)_{f 2}\right]\right\} .
$$

Using Eqs. (C11), (D6) and (D7), the integral over $z_{1}$ in Eq. (F3) equals

$$
\begin{aligned}
& \int_{z_{1}} \prod_{f=1}^{N_{f}}\left\{\left[\left(\mathcal { u } _ { f 1 } J _ { f 1 } ( x _ { f 1 } ) ( \mathcal { u } _ { f 1 } ] \left[(\underbrace{}_{f 2} J_{f 2}\left(x_{f 2}\right)(\underbrace{}_{f 2}]\}\right.\right.\right.\right. \\
& =\int_{z_{1}} \prod_{f=1}^{N_{f}}\left[\psi_{f 1}^{\dagger}\left(x_{f 1}, z_{1}\right) J_{1}\left(x_{f 1}\right) \psi_{f 1}\left(x_{f 1}, z_{1}\right)\right]\left[\widehat{\psi}_{f 1}^{\dagger}\left(x_{f 2}, z_{1}\right) J_{2}\left(x_{f 2}\right) \widehat{\psi}_{f 1}\left(x_{f 2}, z_{1}\right)\right] \\
& =\int \prod_{z_{1}}^{N_{f}}\left\{\left[\rho_{1}^{3} \mathcal{F}_{1}\left(x_{f 1} ; z_{1}, \rho_{1}, U_{1}\right)\right]\left[\rho_{1}^{2} \rho_{2} \mathcal{F}_{2}\left(x_{f 2} ; z_{1}, \rho_{1}, U_{2}\right)\right]\right\},
\end{aligned}
$$

and similarly for Eq. (F4). The overlap integral for any $N_{f}$ is given by

$$
\mathcal{I}_{N_{f}, i}\left(\rho_{1}, \rho_{2},\left\{x_{f i}\right\}\right)=\int \prod_{z_{i}}^{N_{f}} \mathbb{X}_{i=1}\left(x_{f i}, z_{i}\right)^{2}=\left(\rho_{1} \rho_{2}\right)^{2 N_{f}} \int d^{4} z_{i} \prod_{f=1}^{N_{f}} \frac{\left(x_{f i}-z_{i}\right)^{2}}{\left[\left(x_{f i}-z_{i}\right)^{2}+\rho_{i}^{2}\right]^{3}}
$$

In general this integral depends on the locations of the two sources. For an instanton with $Q=2$, though, if the constituentinstantons are small, then the zero modes $\psi_{f 2}^{(2)}(x)$ generate the overlap term in Eq. (F3). This overlap stems from configurations where $\psi_{f 2}^{(2)}\left(x_{f 2}\right)$ is closer the first constituent-instanton, i.e., $\left|x_{f 2}-z_{1}\right| \ll\left|x_{f 2}-z_{2}\right| \approx\left|z_{1}-z_{2}\right|=\left|R_{12}\right|$. Then,

$$
\left.\psi_{f 2}^{(2)}\left(x_{f 2}\right)\right|_{\left|x_{f 2}-z_{1}\right| \ll\left|x_{f 2}-z_{2}\right|} \approx-\hat{\psi}_{f 1}\left(x_{f 2}, z_{1}\right) \frac{\rho_{1} \rho_{2}\left|R_{12}\right|}{\left(R_{12}^{2}+\rho_{2}^{2}\right)^{3 / 2}}
$$

This limit is consistent with Eqs. (C9) and (C10) as long as $\left|x_{f 2}-z_{1}\right| \gg \rho_{1}, \rho_{2}$. Other terms $\sim \mathcal{V}^{2}$ are dominated by configurations, where at least one of the zero modes $\psi_{f 2}^{(2)}\left(x_{f 2}\right)$ is close to $z_{2}$, and are suppressed for $\left|x_{f 2}-z_{1}\right| \ll\left|x_{f 2}-z_{2}\right|$. The analogous statement is true for the overlap from $\psi_{f 1}^{(2)}\left(x_{f 1}\right)$ in Eq. (F4). Hence, in this case the overlap term only depends on the instanton size and the distance between the instantons,

$$
\mathbb{X}_{i}=\frac{\rho_{1} \rho_{2}\left|R_{12}\right|}{\left(R_{12}^{2}+\rho_{i}^{2}\right)^{3 / 2}},
$$

and the quark zero mode determinant is dominated by a term $\sim \mathcal{V}$. The overlap integral for $N_{f} \geq 2$ becomes 


$$
\begin{aligned}
\mathcal{I}_{N_{f}, i}\left(\rho_{1}, \rho_{2}\right) & =\left(\rho_{1} \rho_{2}\right)^{2 N_{f}} \int d^{4} R_{12} \prod_{f=1}^{N_{f}} \frac{R_{12}^{2}}{\left(R_{12}^{2}+\rho_{i}^{2}\right)^{3}}=\left(\rho_{1} \rho_{2}\right)^{2 N_{f}} \int d^{4} R_{12} \frac{R_{12}^{2 N_{f}}}{\left(R_{12}^{2}+\rho_{i}^{2}\right)^{3 N_{f}}} \\
& =\frac{\pi^{2}\left(N_{f}+1\right) !\left(2 N_{f}-3\right) !}{\left(3 N_{f}-1\right) !}\left(\frac{\rho_{1} \rho_{2}}{\rho_{i}^{2}}\right)^{2 N_{f}} \rho_{i}^{4} .
\end{aligned}
$$

For any $N_{f}$, the partition function for $Q=2$ is

$$
\left.Z^{(2)}[J]\right|_{\text {local }}=\int d \rho_{1} n_{1}\left(\rho_{1}\right) \rho_{1}^{5 N_{f}} \int d \rho_{2} n_{1}\left(\rho_{2}\right) \rho_{2}^{N_{f}} \mathcal{I}_{N_{f}, 2} \int d^{4} z_{1} \prod_{i=1}^{2}\left\{\int d U_{i} \prod_{f=1}^{N_{f}} \int d^{4} x_{f i} \mathcal{F}_{i}\left(x_{f i} ; z_{1}, U_{i}\right)\right\}
$$

with $\mathcal{F}_{i}$ defined in Eq. (D6). We emphasize that since at large distances $\mathcal{F}_{i}$ contains two free quark propagators (D8), the generating functional is that of a correlation function between $2 N_{f} Q$ quarks.

\section{APPENDIX G: THE EFFECTIVE INTERACTION}

We now derive the effective action from the quark zero mode determinant computed in the previous sections. The main trick is to exploit the fact that far away from the instanton, the determinant can be expressed in terms of free quark propagators, cf. Eq. (D8), so that it mimics an operator without a background field, located at the position of the instanton.

\section{Any topological charge at leading order}

Before we discuss the local interaction for $Q=2$, we consider the result to leading order for a small $Q$-instanton, under the ansatz,

$$
\begin{aligned}
Z_{\mathrm{eff}, \mathrm{LO}}^{(Q)+}[\bar{J}]= & \int \mathcal{D} \psi \mathcal{D} \bar{\psi} \exp \left\{-S[\chi]+\int d^{4} x \bar{\psi} \bar{J} \psi\right\} V_{\mathrm{eff}, \mathrm{LO}}^{(Q)+}, \\
V_{\mathrm{eff}, \mathrm{LO}}^{(Q)+}= & \frac{\kappa_{Q, \mathrm{LO}}}{K_{Q, N_{f}}} \prod_{i=1}^{Q} \\
& \times\left\{\int d^{4} z_{i} \int d U_{i} \prod_{f=1}^{N_{f}}\left[\bar{\psi}_{f}\left(z_{i}\right) \omega_{i}\right]\left[\bar{\omega}_{i} \psi_{f}\left(z_{i}\right)\right]\right\},
\end{aligned}
$$

where again without loss of generality we assume $Q$ is positive. This ansatz applies only to leading order (LO) for small constituent-instantons. $\omega_{i}$ are constant tensors carrying spin and color which are determined below, and $K_{Q, N_{f}}$ is defined in Eq. (G4). The preexponential factor $V_{\text {eff,LO }}^{(Q)+}$ generates a nonlocal $2 N_{f} Q$-correlation function with coupling strength $\kappa_{Q}$. The superscript ${ }^{+}$indicates that this is contribution from instantons; - denotes that from antiinstantons. Because of Fermi statistics, this term can be rewritten as a determinant,

$$
\prod_{f=1}^{N_{f}}\left(\bar{\psi}_{f} \omega_{i}\right)\left(\bar{\omega}_{i} \psi_{f}\right)=\frac{1}{N_{f} !} \operatorname{det}_{f g}\left[\left(\bar{\psi}_{f} \omega_{i}\right)\left(\bar{\omega}_{i} \psi_{g}\right)\right] .
$$

This explains why we could take the quark source $J$ to be diagonal in color and flavor, as all other contributions are given by permutations of the quark fields. The correlation function generated by $V_{\mathrm{eff}, \mathrm{LO}}^{(Q)+}$ can be computed by expressing the exponential as a power series in $\bar{J}$,

$$
\begin{aligned}
e^{-S+\int_{x} \bar{\psi} \bar{J} \psi}= & e^{-S}\left\{1+\int_{x_{11}} \bar{\psi}\left(x_{11}\right) \bar{J}\left(x_{11}\right) \psi\left(x_{11}\right)\right. \\
& +\frac{1}{2} \int_{x_{11}} \bar{\psi}\left(x_{11}\right) \bar{J}\left(x_{11}\right) \psi\left(x_{1}\right) \\
& \times \int_{x_{12}} \bar{\psi}\left(x_{12}\right) \bar{J}\left(x_{12}\right) \psi\left(x_{12}\right) \\
& +\cdots+\frac{1}{\left(N_{f} Q\right) !} \prod_{i=1}^{Q} \prod_{f=1}^{N_{f}} \\
& \left.\times \int_{x_{f i}} \bar{\psi}\left(x_{f i}\right) \bar{J}\left(x_{f i}\right) \psi\left(x_{f i}\right)+\ldots\right\} .
\end{aligned}
$$

Wick's theorem is then used to contract the quarks from the sources with those in $V_{\mathrm{eff}, \mathrm{LO}}^{(Q)+}$. Note our suggestive notation for the vertex locations in Eq. (G1) and the locations of the sources in Eq. (G3). For small constituent-instantons, the $z_{i}$ in Eq. (G1) are by definition widely separated. As a result, contractions of quark fields are suppressed except for those where all quarks sourced at $x_{f i}$ are contracted at $z_{j}$ in $V_{\text {eff,LO }}^{(Q)+}$. Any other contraction involves at least one propagator $\Delta\left(z_{i}, z_{j}\right)$, with $i \neq j$, which is suppressed for small constituent-instantons. Hence, only the term of order $N_{f} Q$ in $\bar{J}$ in Eq. (G3) contributes. For fixed $f$, there are $Q$ ! equivalent ways to contract the quarks at $x_{f i}$ with the ones at $z_{j}$. Since this can be done for each $f$, there are $(Q !)^{N_{f}}$ equivalent contributions. All other contractions are suppressed, since they contain at least one $\Delta\left(z_{i}, z_{j}\right)$. We combine this into the combinatorial factor, 


$$
K_{Q, N_{f}}=\frac{(Q !)^{N_{f}}}{\left(N_{f} Q\right) !}
$$

Expanding the exponential in powers of the sources and using Wick's theorem for small constituent-instantons, $Z_{\text {eff,LO }}^{(Q)+}[\bar{J}]$ is dominated by a $2 N_{f} Q$-quark correlation function multiplied by $K_{Q, N_{f}}$. To compensate for this factor, we introduced a factor of $1 / K_{Q, N_{f}}$ for the effective coupling in Eq. (G1). In all we find

$$
\begin{aligned}
& \left\langle\frac{1}{\left(N_{f} Q\right) !} \prod_{i=1}^{Q} \prod_{f=1}^{N_{f}}\left[\int_{x_{f i}} \bar{\psi}\left(x_{f i}\right) \bar{J}\left(x_{f i}\right) \psi\left(x_{f i}\right)\right] V_{\mathrm{eff}, \mathrm{LO}}^{(Q)+}\right\rangle \\
& =\kappa_{Q, \mathrm{LO}} \prod_{i=1}^{Q} \int_{z_{i}} \int d U_{i} \prod_{f=1}^{N_{f}} \\
& \quad \times \int_{x_{f i}} \bar{\omega}_{i} \Delta\left(x_{f i}-z_{i}\right) \bar{J}\left(x_{f i}\right) \Delta\left(x_{f i}-z_{i}\right) \omega_{i} .
\end{aligned}
$$

Comparing this with Eq. (D10), using Eq. (D8), we find

$$
\begin{aligned}
\kappa_{Q, \mathrm{LO}} & \prod_{i=1}^{Q} \int d^{4} z_{i} \int d U_{i} \prod_{f=1}^{N_{f}} \\
& \times \int d^{4} x_{f i} \bar{\omega}_{i} \Delta\left(x_{f i}-z_{i}\right) \bar{J}\left(x_{f i}\right) \Delta\left(x_{f i}-z_{i}\right) \omega_{i} \\
= & \frac{\left(8 \pi^{2}\right)^{N_{f} Q}}{Q !}\left[\int d \rho_{i} n_{1}\left(\rho_{i}\right) \rho_{i}^{3 N_{f}}\right]^{Q} \prod_{i=1}^{Q} \int d^{4} z_{i} \int d U_{i} \prod_{f=1}^{N_{f}} \\
& \times \int d^{4} x_{f i} U_{i}^{\dagger} \varphi_{R}^{\dagger} \Delta\left(x_{f i}-z_{i}\right) \bar{J}\left(x_{f i}\right) \Delta\left(x_{f i}-z_{i}\right) \varphi_{R} U_{i} .
\end{aligned}
$$

From this, the effective coupling is

$$
\kappa_{Q, \mathrm{LO}}=\frac{1}{Q !}\left[\left(8 \pi^{2}\right)^{N_{f}} \int d \rho_{i} n_{1}\left(\rho_{i}\right) \rho_{i}^{3 N_{f}}\right]^{Q} .
$$

Aside from the combinatoric factor, this is precisely the effective coupling derived in [4] for the single instanton to the $Q^{\text {th }}$ power. From the integrands on both sides of Eq. (G6) we infer that the tensor $\omega$ obeys the identity,

$$
\left(\omega_{i}\right)_{\alpha}^{a}\left(\bar{\omega}_{i}\right)_{\beta}^{a}=\varphi_{R}^{\alpha b} U_{i}^{b a}\left(U_{i}^{\dagger}\right)^{a c}\left(\varphi_{R}^{\dagger}\right)^{c \beta},
$$

where the color indices $(a, b, c)$ and spinor indices $(\alpha, \beta)$ are explicit here. The color structure of $\omega_{i}$ is fixed by requiring that it carries the global color orientation $U_{i}$,

$$
\left(U_{i}\right)^{a b} \omega_{\alpha}^{b}=\left(\omega_{i}\right)_{\alpha}^{a}
$$

Further, from the explicit form of the spinor $\varphi_{R}$ in Eq. (C7),

$$
\varphi_{R}^{\alpha a}\left(\varphi_{R}^{\dagger}\right)^{a \beta}=\mathbb{P}_{R}^{\alpha \beta},
$$

where $\mathbb{P}_{R}$ is the right-handed projection operator defined in Eq. (A4). This implies

$$
\omega_{\alpha}^{a} \bar{\omega}_{\beta}^{a}=\mathbb{P}_{R}^{\alpha \beta}
$$

The integration over the orientation in the gauge group in Eq. (G1) can now be carried out. Since we have the same integral for different topological charge indices $i$, the integral for fixed $i$ is done following Refs. [4]. The final result is this result to the $Q^{\text {th }}$ power,

$$
\begin{aligned}
& \int d U_{i} \prod_{f=1}^{N_{f}}\left[\bar{\psi}_{f}\left(z_{i}\right) \omega_{i}\right]\left[\bar{\omega}_{i} \psi_{f}\left(z_{i}\right)\right] \\
& =\int d U_{i} \prod_{f=1}^{N_{f}}\left[\bar{\psi}_{f}\left(z_{i}\right) U_{i} \omega\right]\left[\bar{\omega} U_{i}^{\dagger} \psi_{f}\left(z_{i}\right)\right] .
\end{aligned}
$$

For a $S U\left(N_{c}\right)$ gauge group,

$$
\int d U_{i} \prod_{f=1}^{N_{f}} U_{i}^{a_{f} b_{f}}\left(U_{i}^{\dagger}\right)^{c_{f} d_{f}},
$$

where $U_{i}$ is an element of $S U\left(N_{c}\right) / \mathcal{I}_{N_{c}}$, with $\mathcal{I}_{N_{c}}$ the stability group of the instanton, the set of $S U\left(N_{c}\right)$ transformations that leave the instanton unchanged. For $N_{c}=2$ this is the identity, and $d U_{i}$ is the corresponding Haar measure. For arbitrary $N_{f}$ and $N_{c}$ the integration is more involved. In general, the group integration in Eq. (G12) yields products of terms which are color singlet, $\sim \bar{\psi}_{L} \psi_{R}$, and nonsinglet, $\sim \psi_{L} \psi_{L}$. In vacuum only the color singlet terms, $\sim \bar{\psi}_{L} \psi_{R}$, are important, but at nonzero density, the nonsinglet terms $\sim \psi_{L} \psi_{L}$ affect color superconductivity. For two flavors, product of color singlet terms is extracted from

$$
\int d U_{i} U_{i}^{a_{1} b_{1}}\left(U_{i}^{\dagger}\right)^{c_{1} d_{1}} U_{i}^{a_{2} b_{2}}\left(U_{i}^{\dagger}\right)^{c_{2} d_{2}}=c_{N_{c}}\left(\delta^{a_{1} d_{1}} \delta^{a_{2} d_{2}} \delta^{b_{1} c_{1}} \delta^{b_{2} c_{2}}+\delta^{a_{1} d_{2}} \delta^{a_{2} d_{1}} \delta^{b_{1} c_{2}} \delta^{b_{2} c_{1}}\right)+\text { (nonsinglet) }
$$

$c_{N_{c}}$ is a $N_{c}$-dependent constant. We find 


$$
\begin{aligned}
& \left.\int d U_{i} \prod_{f=1}^{N_{f}}\left[\bar{\psi}_{f}\left(z_{i}\right) U_{i} \omega\right]\left[\bar{\omega} U_{i}^{\dagger} \psi_{f}\left(z_{i}\right)\right]\right|_{\text {singlet }} \\
& =c_{N_{c}}\left(\delta^{a_{1} d_{1}} \delta^{a_{2} d_{2}} \delta^{b_{1} c_{1}} \delta^{b_{2} c_{2}}+\delta^{a_{1} d_{2}} \delta^{a_{2} d_{1}} \delta^{b_{1} c_{2}} \delta^{b_{2} c_{1}}\right)\left[\bar{\psi}_{1}^{\alpha a_{1}}\left(z_{i}\right) \omega_{\alpha}^{b_{1}} \bar{\omega}_{\beta}^{c_{1}} \psi_{1}^{\beta d_{1}}\left(z_{i}\right) \bar{\psi}_{2}^{\alpha a_{2}}\left(z_{i}\right) \omega_{\alpha}^{b_{2}} \bar{\omega}_{\beta}^{c_{2}} \psi_{1}^{\beta d_{2}}\left(z_{i}\right)\right] \\
& =c_{N_{c}}\left[\bar{\psi}_{1}^{\alpha a_{1}}\left(z_{i}\right) \omega_{\alpha}^{b_{1}} \bar{\omega}_{\beta}^{b_{1}} \psi_{1}^{\beta a_{1}}\left(z_{i}\right) \bar{\psi}_{2}^{\alpha a_{2}}\left(z_{i}\right) \omega_{\alpha}^{b_{2}} \bar{\omega}_{\beta}^{b_{2}} \psi_{2}^{\beta a_{2}}\left(z_{i}\right)+\bar{\psi}_{1}^{\alpha a_{1}}\left(z_{i}\right) \omega_{\alpha}^{b_{1}} \bar{\omega}_{\beta}^{b_{2}} \psi_{1}^{\beta a_{2}}\left(z_{i}\right) \bar{\psi}_{2}^{\alpha a_{2}}\left(z_{i}\right) \omega_{\alpha}^{b_{2}} \bar{\omega}_{\beta}^{b_{1}} \psi_{2}^{\beta a_{1}}\left(z_{i}\right)\right] \\
& =c_{N_{c}}\left[\bar{\psi}_{1}^{\alpha a_{1}}\left(z_{i}\right) \omega_{\alpha}^{b_{1}} \bar{\omega}_{\beta}^{b_{1}} \psi_{1}^{\beta a_{1}}\left(z_{i}\right) \bar{\psi}_{2}^{\alpha a_{2}}\left(z_{i}\right) \omega_{\alpha}^{b_{2}} \bar{\omega}_{\beta}^{b_{2}} \psi_{2}^{\beta a_{2}}\left(z_{i}\right)-\bar{\psi}_{1}^{\alpha a_{1}}\left(z_{i}\right) \omega_{\alpha}^{b_{1}} \bar{\omega}_{\beta}^{b_{1}} \psi_{2}^{\beta a_{1}}\left(z_{i}\right) \bar{\psi}_{2}^{\alpha a_{2}}\left(z_{i}\right) \omega_{\alpha}^{b_{2}} \bar{\omega}_{\beta}^{b_{2}} \psi_{1}^{\beta a_{2}}\left(z_{i}\right)\right] .
\end{aligned}
$$

Now we can apply the identity for $\omega$ in Eq. (G11) to arrive at,

$$
\begin{aligned}
\int & \left.d U_{i} \prod_{f=1}^{N_{f}}\left[\bar{\psi}_{f}\left(z_{i}\right) U_{i} \omega\right]\left[\bar{\omega} U_{i}^{\dagger} \psi_{f}\left(z_{i}\right)\right]\right|_{\text {singlet }} \\
= & c_{N_{c}}\left[\bar{\psi}_{1}\left(z_{i}\right) \mathbb{P}_{R} \psi_{1}\left(z_{i}\right) \bar{\psi}_{2}\left(z_{i}\right) \mathbb{P}_{R} \psi_{2}\left(z_{i}\right)\right. \\
& \left.-\bar{\psi}_{1}\left(z_{i}\right) \mathbb{P}_{R} \psi_{2}\left(z_{i}\right) \bar{\psi}_{2}\left(z_{i}\right) \mathbb{P}_{R} \psi_{1}\left(z_{i}\right)\right] \\
= & c_{N_{c}} \operatorname{det}_{f g}\left[\bar{\psi}_{f}\left(z_{i}\right) \mathbb{P}_{R} \psi_{g}\left(z_{i}\right)\right] .
\end{aligned}
$$

Plugging this into Eq. (G1), we find

$$
\left.V_{\text {eff }, \mathrm{LO}}^{(Q)+}\right|_{\text {singlet }}=\frac{\kappa_{Q, \mathrm{LO}}}{K_{Q, N_{f}}} \prod_{i=1}^{Q} \int d^{4} z_{i} \operatorname{det}_{f g}\left[\bar{\psi}_{f}\left(z_{i}\right) \mathbb{P}_{R} \psi_{g}\left(z_{i}\right)\right],
$$

with the coupling $\kappa_{Q, \text { LO }}$ given in Eq. (G7). For any number of flavors, the color singlet channel is a determinant in flavor, since the structure of the gauge group integration in Eq. (G14) is

$$
\begin{aligned}
& \int d U \prod_{i} U^{a_{i} b_{i}}\left(U^{\dagger}\right)^{c_{i} d_{i}} \\
& =c_{N_{c} N_{f}} \sum_{\sigma} \prod_{i} \delta_{a_{i} d_{\sigma(i)}} \delta_{b_{i} c_{\sigma(i)}}+\text { (nonsinglet) }
\end{aligned}
$$

where $\sigma(i)$ are permutations of $i=1, \ldots, N_{f}$; see e.g., [53].

To obtain the effective action we need to exponentiate $V_{\text {eff.LO }}^{(Q)+}$. So far we considered the generating functional in the background of a single $Q$-instanton in the limit of small constituent instantons. For a single $Q$-anti-instanton we replace the right-handed with the left-handed projector operator, $\mathbb{P}_{R} \rightarrow \mathbb{P}_{L}$, in Eq. (G17) to obtain $V_{\text {eff,LO }}^{(Q)-}$. We now assume that the field configurations of topological charge $Q$ are described by a dilute gas of $Q$-instantons and antiinstantons. This generalizes the dilute instanton gas in [4] to arbitrary topological charge.

In general, the complete contribution of $Q$-instantons to the functional integral of a dilute instanton gas is a simple statistical ensemble,

$$
\begin{aligned}
& \sum_{\nu_{+}=1}^{\infty} \sum_{\nu_{-}=1}^{\infty} \frac{\left(\kappa_{Q} / K_{Q, N_{f}}\right)^{\nu_{+}+\nu_{-}}}{\nu_{+} ! \nu_{-} !}\left(V_{\mathrm{eff}}^{(Q)+}\right)^{\nu_{+}}\left(V_{\mathrm{eff}}^{(Q)-}\right)^{\nu_{-}} \\
& =\exp \left[\frac{\kappa_{Q}}{K_{Q, N_{f}}}\left(V_{\mathrm{eff}}^{(Q)+}+V_{\mathrm{eff}}^{(Q)-}\right)\right]
\end{aligned}
$$

$\nu_{+}$and $\nu_{-}$are the numbers of instantons and anti-instantons. The anomalous contribution to the effective action is

$$
\Delta S_{\mathrm{eff}, \mathrm{LO}}^{(Q)}=-\frac{\kappa_{Q}}{K_{Q, N_{f}}}\left(V_{\mathrm{eff}}^{(Q)+}+V_{\mathrm{eff}}^{(Q)-}\right)
$$

However, since the leading-oder contribution of a $Q$ instanton factorizes into single-instanton contributions, a dilute gas of single-instantons already fully captures this contribution. Only genuine multi-instanton configurations, where the constituent-instantons overlap to some extent, contribute to multi-instanton corrections to the conventional dilute instanton gas. Such contributions have been computed in Appendixes E and F, and the effective action which results discussed in the next section. A detailed discussion of the dilute multi-instanton gas can be found in [51].

In principle, instantons of any topological charge contribute to the functional integral. Of course, in the semiclassical regime the contributions with higher topological charge are exponentially suppressed due to the factor $\exp \left(-8 \pi^{2} Q / g^{2}\right)$ in the instanton density. This picture is therefore not in conflict with lattice results on the topological charge at large temperature [34-36]. Still, these contributions can be present and the resulting anomalous contribution to the effective action of a dilute gas of instantons and anti-instantons of all topological charges is

$$
\Delta S_{\mathrm{eff}}=\sum_{Q} \Delta S_{\mathrm{eff}}^{(Q)}
$$

While the effective interactions are certainly small in the dilute instanton gas, they might have relevant phenomenological implications as they manifest in anomalous correlation functions of higher order. 


\section{The local interaction for $Q=2$}

We now repeat the analysis for $Q=2$, taking into account the results of Appendixes E and F. Instead of $\sim \mathcal{V}^{2}$ in the spacetime volume $\mathcal{V}$, these are $\sim \mathcal{V}$. We take

$$
\begin{aligned}
Z_{\mathrm{eff}}^{(2)+}[\bar{J}] & =\int \mathcal{D} \psi \mathcal{D} \bar{\psi} \exp \left\{-S[\chi]+\int_{x} \bar{\psi} \bar{J} \psi\right\} V_{\mathrm{eff}}^{(2)+}, \\
V_{\mathrm{eff}}^{(2)+} & =\frac{\kappa_{2}}{K_{2, N_{f}}} \int d^{4} z \prod_{i=1}^{2}\left\{\int d U_{i} \prod_{f=1}^{N_{f}}\left[\bar{\psi}_{f}(z) \omega_{i}\right]\left[\bar{\omega}_{i} \psi_{f}(z)\right]\right\} .
\end{aligned}
$$

Following the previous analysis, averaging over $V_{\mathrm{eff}}^{(2)+}$ gives

$$
\begin{aligned}
\left\langle\frac{1}{\left(2 N_{f}\right) !} \prod_{i=1}^{2} \prod_{f=1}^{N_{f}}\left[\int_{x_{f i}} \bar{\psi}\left(x_{f i}\right) \bar{J}\left(x_{f i}\right) \psi\left(x_{f i}\right)\right] V_{\mathrm{eff}}^{(2)+}\right\rangle= & \kappa_{2} \int d^{4} z \prod_{i=1}^{2} \int d U_{i} \prod_{f=1}^{N_{f}} \int d^{4} x_{f i} \bar{\omega}_{i} \Delta\left(x_{f i}-z\right) \\
& \times \bar{J}\left(x_{f i}\right) \Delta\left(x_{f i}-z\right) \omega_{i} .
\end{aligned}
$$

Choosing $\kappa_{2}$ and the tensors $\omega$ so that this correlation function is identical to the generating functional for $Q=2$ in Eq. (F10),

$$
\begin{aligned}
\kappa_{2} \int & d^{4} z \prod_{i=1}^{2} \int d U_{i} \prod_{f=1}^{N_{f}} \int d^{4} x_{f i} \bar{\omega}_{i} \Delta\left(x_{f i}-z\right) \bar{J}\left(x_{f i}\right) \Delta\left(x_{f i}-z\right) \omega_{i} \\
= & \left(8 \pi^{2}\right)^{2 N_{f}} \int d \rho_{2} n_{1}\left(\rho_{2}\right) \rho_{2}^{N_{f}} \int d \rho_{1} n_{1}\left(\rho_{1}\right) \rho_{1}^{5 N_{f}} \mathcal{I}_{N_{f}, 2} \\
& \times \int d^{4} z \prod_{i=1}^{2}\left\{\int d U_{i} \prod_{f=1}^{N_{f}} \int d^{4} x_{f i} U_{i}^{\dagger} \varphi_{R}^{\dagger} \Delta\left(x_{f i}-z\right) \bar{J}\left(x_{f i}\right) \Delta\left(x_{f i}-z\right) \varphi_{R} U_{i}\right\},
\end{aligned}
$$

which is valid for any $N_{f}$. The overlap integral $\mathcal{I}_{1}$ is given by Eq. (E6) and $\mathcal{I}_{N_{f}}$ for $N_{f} \geq 2$ by Eq. (F9). From this we infer

$$
\kappa_{2}=\left(8 \pi^{2}\right)^{2 N_{f}} \int d \rho_{2} n_{1}\left(\rho_{2}\right) \rho_{2}^{N_{f}} \int d \rho_{1} n_{1}\left(\rho_{1}\right) \rho_{1}^{5 N_{f}} \mathcal{I}_{N_{f}, 2}
$$

The determination of $\omega$ and the integration over the gauge group are as before. The main difference here is that all propagators connect to the same point $z$. For the channel which is a product of color singlet operators,

$$
\left.V_{\mathrm{eff}}^{(2)+}\right|_{\text {singlet }}=\int_{z} \frac{\kappa_{2}}{K_{2, N_{f}}}\left[\operatorname{det}_{f g}\left(\bar{\psi}_{f}(z) \mathbb{P}_{R} \psi_{g}(z)\right)\right]^{2} .
$$

This is a genuine 2-instanton contribution. As discussed in the previous section, a dilute gas of 2-instantons then leads to

$$
\Delta S_{\mathrm{eff}}^{(2)}=-\int d^{4} x \frac{\kappa_{2}}{K_{2, N_{f}}}\left\{\left[\operatorname{det}_{f g}\left(\bar{\psi}_{f}(x) \mathbb{P}_{R} \psi_{g}(x)\right)\right]^{2}+\left[\operatorname{det}_{f g}\left(\bar{\psi}_{f}(x) \mathbb{P}_{L} \psi_{g}(x)\right)\right]^{2}\right\},
$$

with $\kappa_{2}$ in Eq. (G25) and $K_{2, N_{f}}=2^{N_{f}} /\left(2 N_{f}\right) !$

\section{APPENDIX H: A LOW-ENERGY MODEL}

We use our results to construct a linear sigma model (LSM) for two flavors. For the sake of generality we include one more anomalous quartic term which is generated by instantons with $Q=1$; we set it to zero in the main text. The anomalous part of the effective Lagrangian is 


$$
\begin{aligned}
\mathcal{L}_{\mathcal{G}_{\mathrm{A}}}= & -\chi_{1}\left(\operatorname{det} \Phi+\operatorname{det} \Phi^{\dagger}\right)-\chi_{2}\left[(\operatorname{det} \Phi)^{2}\right. \\
& \left.+\left(\operatorname{det} \Phi^{\dagger}\right)^{2}\right]+\bar{\lambda}_{3}\left(\operatorname{Tr} \Phi^{\dagger} \Phi\right)\left(\operatorname{det} \Phi+\operatorname{det} \Phi^{\dagger}\right) .
\end{aligned}
$$

We set $\bar{\lambda}_{3}=0$ in the main text. The meson field is given by

$$
\Phi=(\sigma+i \eta)+\left(\vec{a}_{0}+i \vec{\pi}\right) \vec{\tau} .
$$

The equations of motion are

$$
\left.\frac{\delta \Gamma}{\delta \phi}\right|_{\phi=\bar{\phi}}=0
$$

$\phi=\left(\sigma, \vec{a}_{0}, \eta, \vec{\pi}\right)$, with the vacuum expectation value $\bar{\phi}=(\bar{\sigma}, \overrightarrow{0}, 0, \overrightarrow{0})$,

$$
\bar{\sigma}^{2}=\frac{-2\left(m^{2}-\chi_{1}\right)}{\lambda_{1}+2 \lambda_{2}+2 \bar{\lambda}_{3}-\chi_{2}} .
$$

When $m^{2}>\chi_{1}$ the expectation value of $\phi$ vanishes. When $m^{2}<\chi_{1} G_{\text {qu }}$ spontaneously breaks to $S U_{V}(2) \times \mathbb{Z}_{2}^{A}$. If there were no anomalous terms, $\mathcal{L}_{\mathcal{G}_{\mathrm{qu}}}=0, U_{A}(1)$ would also break, resulting in four Goldstone bosons $\vec{\pi}$ and $\eta$. In the presence of the anomalous terms $U_{A}(1)$ is broken explicitly, and only pions are massless. Due to isospin symmetry, there are four distinct masses,

$$
\begin{aligned}
& m_{\sigma}^{2}=m^{2}+\chi_{1}-\frac{3}{2}\left(\lambda_{1}+2 \lambda_{2}+2 \bar{\lambda}_{3}-\chi_{2}\right) \bar{\sigma}^{2} \\
& m_{\pi}^{2}=m^{2}+\chi_{1}-\frac{1}{2}\left(\lambda_{1}+2 \lambda_{2}+2 \bar{\lambda}_{3}-\chi_{2}\right) \bar{\sigma}^{2} \\
& m_{\eta}^{2}=m^{2}-\chi_{1}-\frac{1}{2}\left(\lambda_{1}+2 \lambda_{2}+3 \chi_{2}\right) \bar{\sigma}^{2} \\
& m_{a_{0}}^{2}=m^{2}-\chi_{1}-\frac{1}{2}\left(3 \lambda_{1}+2 \lambda_{2}+\chi_{2}\right) \bar{\sigma}^{2}
\end{aligned}
$$

In the symmetric phase, $\bar{\sigma}=0$, only the quadratic terms contribute to the masses directly and the $Q=1$ term $\chi_{1}$ induces a splitting of the chiral pairs $(\sigma, \vec{\pi})$ and $\left(\eta, \vec{a}_{0}\right)$. In the symmetric phases higher order couplings only influence these masses via loop corrections. Inserting the expectation values from Eq. (H4),

$$
\begin{aligned}
& m_{\pi}^{2}=0 \\
& m_{\sigma}^{2}=-2\left(m^{2}-\chi_{1}\right) \\
& m_{\eta}^{2}=\frac{-2 m^{2}\left(2 \chi_{2}-\bar{\lambda}_{3}\right)+2 \chi_{1}\left(\lambda_{1}+2 \lambda_{2}+\bar{\lambda}_{3}+\chi_{2}\right)}{\lambda_{1}+2 \lambda_{2}+2 \bar{\lambda}_{3}-\chi_{2}}, \\
& m_{a_{0}}^{2}=\frac{-2 m^{2}\left(\lambda_{1}+\chi_{2}-\bar{\lambda}_{3}\right)+2 \chi_{1}\left(2 \lambda_{1}+2 \lambda_{2}+\bar{\lambda}_{3}\right)}{\lambda_{1}+2 \lambda_{2}+2 \bar{\lambda}_{3}-\chi_{2}} .
\end{aligned}
$$

The pion is always a Goldstone boson. To explore the influence of the anomalous terms, we fix the masses in vacuum using the following observables:

$$
\begin{aligned}
f_{\pi} & =\bar{\sigma}_{0}=93 \mathrm{MeV}, \quad m_{\sigma, 0}=400 \mathrm{MeV}, \\
m_{\eta, 0} & =820 \mathrm{MeV}, \quad m_{a_{0}, 0}=980 \mathrm{MeV} .
\end{aligned}
$$

The $\eta$ mass is taken from Ref. [32]. For the other masses, we chose values compatible with Ref. [49]. Note that we identify the $\sigma$ meson with the $f_{0}(500)$. Within the meanfield approximation, and in absence of effects from topological charge $Q>1$, taking $\bar{\lambda}_{3}=0$ all parameters, including $\chi_{1}$, are fixed by the vacuum masses. This then also fixes the amount of axial symmetry breaking above the chiral phase transition, as the only anomalous contribution to the masses in the symmetric phase stems from $\chi_{1}$. When $\chi_{2} \neq 0$ the vacuum mass spectrum can be fixed for different values for $\chi_{2}$, and we can explore the influence of interactions induced by higher topological charge on the mass spectrum.

For a given value of $\chi_{1}$, the value of $m^{2}$ determines whether the symmetry is broken or not. Thus in mean field theory varying temperature is equivalent to changing $m^{2}$. We fix the masses in the vacuum according to Eq. (H7) and study the mass spectrum as a function of $\mathrm{m}^{2}$ for different values of $\chi_{2}$. We then find the following relations:

$$
\begin{aligned}
m^{2} & =\frac{1}{2}\left(m_{\eta}^{2}-m_{\sigma}^{2}\right)-\frac{\bar{\sigma}^{2}}{2}\left(2 \chi_{2}-\bar{\lambda}_{3}\right), \\
\lambda_{1} & =\frac{m_{a_{0}}^{2}-m_{\eta}^{2}}{\bar{\sigma}^{2}}+\chi_{2}, \\
\lambda_{2} & =\frac{m_{\sigma}^{2}+m_{\eta}^{2}-m_{a_{0}}^{2}}{2 \bar{\sigma}^{2}}-\bar{\lambda}_{3}, \\
\chi_{1} & =\frac{1}{2} m_{\eta}^{2}-\frac{\bar{\sigma}^{2}}{2}\left(2 \chi_{2}-\bar{\lambda}_{3}\right) .
\end{aligned}
$$

Since we have six model parameters, but use only four parameters to fix them, we can choose $\chi_{2}$ and $\bar{\lambda}_{3}$ as free parameters. Equation (H8) implies that the anomalous quadratic coupling $\chi_{1}$ is determined by the anomalous quartic couplings $\chi_{2}$ and $\bar{\lambda}_{3}$ via

$$
\chi_{1}=\frac{1}{2} m_{\eta, 0}^{2}-\frac{f_{\pi}^{2}}{2}\left(2 \chi_{2}-\bar{\lambda}_{3}\right)
$$

Setting $\quad \chi_{2}=\bar{\lambda}_{3}=0, \quad$ the coupling $\quad \chi_{1}=m_{\eta, 0}^{2} / 2$. Conversely, if we set $\chi_{1}=\bar{\lambda}_{3}=0$, then the coupling $\chi_{2}=m_{\eta, 0}^{2} /\left(2 f_{\pi}^{2}\right)$. If we use $\chi_{1}$ in the following, we mean $\chi_{1}\left(\chi_{2}, \bar{\lambda}_{3}\right)$ as defined by Eq. (H9).

The system has two characteristic scales in $m^{2}$. The vacuum scale $m_{\mathrm{vac}}^{2}$ is where the masses in the broken phase in Eq. (H6) assume their vacuum values (H7). It can be read off from Eq. (H8),

$$
m_{\mathrm{vac}}^{2}=\chi_{1}-\frac{1}{2} m_{\sigma, 0}^{2}
$$


There is also the critical scale $m_{\text {crit }}^{2}$ for chiral symmetry breaking. It is defined as the value of $m^{2}$ where the expectation value $\bar{\sigma}(\mathrm{H} 4)$ vanishes,

$$
m_{\mathrm{crit}}^{2}=\chi_{1}=m_{\mathrm{vac}}^{2}+\frac{1}{2} m_{\sigma, 0}^{2} .
$$

Hence, the characteristic scales of the system change as $\chi_{2}$ and $\bar{\lambda}_{3}$ vary. To meaningfully compare masses for different values of $\chi_{2}$ and $\bar{\lambda}_{3}$, we define the reduced temperature,

$$
t=\frac{m^{2}-m_{\mathrm{vac}}^{2}}{m_{\mathrm{crit}}^{2}-m_{\mathrm{vac}}^{2}}
$$

and rewrite the masses in terms of $t$,

$$
m^{2}(t)=m_{\mathrm{vac}}^{2}+t\left(m_{\mathrm{crit}}^{2}-m_{\mathrm{vac}}^{2}\right)
$$

$t=0$ is the vacuum and $t=1$ is where the phase transition occurs. As a function of $t$,

$$
\bar{\sigma}^{2}(t)=\left\{\begin{array}{ll}
(1-t) f_{\pi}^{2} & t \leq 1 \\
0 & t>1
\end{array} .\right.
$$

With this parametrization, the masses take very simple forms,

$$
\begin{aligned}
& m_{\sigma}^{2}(t)= \begin{cases}(1-t) m_{\sigma, 0}^{2} & t \leq 1 \\
\frac{1}{2}(t-1) m_{\sigma, 0}^{2} & t>1\end{cases} \\
& m_{\pi}^{2}(t)= \begin{cases}0 & t \leq 1 \\
\frac{1}{2}(t-1) m_{\sigma, 0}^{2} & t>1\end{cases} \\
& m_{\eta}^{2}(t)= \begin{cases}m_{\eta, 0}^{2}-t\left(m_{\eta, 0}^{2}-2 \chi_{1}\right) & t \leq 1 \\
\frac{1}{2}(t-1) m_{\sigma, 0}^{2}+2 \chi_{1} & t>1\end{cases} \\
& m_{a_{0}}^{2}(t)= \begin{cases}m_{a_{0}, 0}^{2}-t\left(m_{a_{0}, 0}^{2}-2 \chi_{1}\right) & t \leq 1 \\
\frac{1}{2}(t-1) m_{\sigma, 0}^{2}+2 \chi_{1} & t>1\end{cases}
\end{aligned}
$$

We see that $m_{\sigma}(t)$ and $m_{\pi}(t)$ are independent of the anomalous couplings. $\sigma$ is the critical mode that becomes massless at the phase transition. The mass splitting between the chiral pairs $(\sigma, \vec{\pi})$ and $\left(\eta, \vec{a}_{0}\right)$ in the symmetric phase is induced by $\chi_{1}$. This mass splitting vanishes in the limit $t \rightarrow \infty$. In absence of the quartic couplings, $\chi_{2}=\bar{\lambda}_{3}=0$, one has $2 \chi_{1}=m_{\eta, 0}^{2}$ so the $\eta$ mass is independent of $t$ in the broken phase. An interesting observation is that for $0<\left(2 \chi_{2}-\bar{\lambda}_{3}\right)<m_{\eta, 0}^{2} / f_{\pi}^{2}, \quad m_{\eta}$ is a strictly decreasing function of $t$ in the broken phase and strictly increasing in the symmetric phase. Hence, it has a minimum at the chiral phase transition. This behavior can therefore be attributed to corrections related to couplings induced by topological charge two.
The masses and the characteristic scales $m_{\mathrm{vac}}$ and $m_{\text {crit }}$ only depend on $\chi_{1}$. Hence, due to Eq. (H9), only the combination of anomalous quartic couplings $\left(2 \chi_{2}-\bar{\lambda}_{3}\right)$ is relevant. We want to focus on the coupling $\sim \chi_{2}$ which is induced by instantons with $Q= \pm 2$. An analysis of the vacuum stability of the effective potential shows that $\bar{\lambda}_{3} \leq m_{\sigma, 0}^{2} / 4 f_{\pi}^{2} \approx 4.62$. We therefore conclude that setting $\bar{\lambda}_{3}=0$, which we made in the main text, is innocuous. Most importantly, Fig. 1 does not change qualitatively for any value of $\bar{\lambda}_{3}$. Our estimated values for the couplings $\chi_{1}$ and $\chi_{2}$ do change, however. The larger $\bar{\lambda}_{3}<0$ is, the smaller $\chi_{1}$ and $\chi_{2}$ become. Even so, $\bar{\lambda}_{3}$ has to become very large to have any significant effect.

By bosonizing the multiquark interactions generated by $Q$-instantons, the fermionic couplings $\kappa_{Q}$ can be related to the anomalous mesonic couplings $\chi_{Q}$. For two flavors, $\kappa_{1}$ is a four-quark coupling and can readily be bosonized by means of a Hubbard-Stratonovich transformation. The 2instanton term $\kappa_{2}$ is an 8-quark interaction for two flavors, so more elaborate path integral bosonization techniques are required [23]. Here we adopt a simplistic bosonization scheme motivated by low-energy models where mesons are coupled to quarks through Yukawa interactions, i.e., quarkmeson models. Using the equations of motion, mesons are proportional to quark bilinears, so we make a simple ansatz based upon Eq. (H2),

$$
\Phi=\frac{1}{2 M^{2}}\left[\left(\bar{\psi} \psi+\bar{\psi} \gamma_{5} \psi\right)+\left(\bar{\psi} \vec{\tau} \psi+\bar{\psi} \gamma_{5} \vec{\tau} \psi\right) \vec{\tau}\right] .
$$

$M$ is a fundamental parameter of our effective theory and has dimensions of mass. By using the identity,

$$
\epsilon^{i j} \epsilon^{f g}=\frac{1}{2}\left[\delta^{i f} \delta^{j g}-\left(\tau^{a}\right)^{i f}\left(\tau^{a}\right)^{j g}\right]
$$

the instanton-induced quark determinant becomes

$$
\begin{aligned}
\operatorname{det}\left(\bar{\psi} \mathbb{P}_{R} \psi\right) & =\frac{1}{2} \epsilon^{i j} \epsilon^{f g}\left(\bar{\psi}_{i} \mathbb{P}_{R} \psi_{f}\right)\left(\bar{\psi}_{j} \mathbb{P}_{R} \psi_{g}\right) \\
& =\frac{1}{8}\left[\left(\bar{\psi} \psi+\bar{\psi} \gamma_{5} \psi\right)^{2}-\left(\bar{\psi} \vec{\tau} \psi+\bar{\psi} \gamma_{5} \vec{\tau} \psi\right)^{2}\right] \\
& =\frac{M^{4}}{2} \operatorname{det} \Phi,
\end{aligned}
$$

and similarly for the anti-instanton term,

$$
\operatorname{det}\left(\bar{\psi} \mathbb{P}_{L} \psi\right)=\frac{M^{4}}{2} \operatorname{det} \Phi^{\dagger}
$$

The 1-instanton induced effective interaction transforms as 


$$
\frac{\kappa_{1}}{K_{1,2}}\left[\operatorname{det}\left(\bar{\psi} \mathbb{P}_{R} \psi\right)+\operatorname{det}\left(\bar{\psi} \mathbb{P}_{L} \psi\right)\right]=\frac{\kappa_{1} M^{4}}{2 K_{1,2}}\left(\operatorname{det} \Phi+\operatorname{det} \Phi^{\dagger}\right),
$$

and for the 2-instanton term,

$$
\begin{gathered}
\frac{\kappa_{2}}{K_{2,2}}\left\{\left[\operatorname{det}\left(\bar{\psi} \mathbb{P}_{R} \psi\right)\right]^{2}+\left[\operatorname{det}\left(\bar{\psi} \mathbb{P}_{L} \psi\right)\right]^{2}\right\} \\
\quad=\frac{\kappa_{2} M^{8}}{4 K_{2,2}}\left[(\operatorname{det} \Phi)^{2}+\left(\operatorname{det} \Phi^{\dagger}\right)^{2}\right] .
\end{gathered}
$$

We therefore identify

$$
\chi_{1}=\frac{\kappa_{1} M^{4}}{2 K_{1,2}}, \quad \chi_{2}=\frac{\kappa_{2} M^{8}}{4 K_{2,2}} .
$$

By plugging this into the expressions for the mesons masses above, the dependence on $\chi_{1}$ and $\chi_{2}$ is replaced by a dependence only on $M$, provided that we know the values of $\kappa_{1}$ and $\kappa_{2}$. This reduces the number of independent parameters to four. Given the four input parameters (H7), at the level of mean field theory the effective Lagrangian of Eq. (11) is uniquely determined.

\section{APPENDIX I: THE INSTANTON DENSITY}

In vacuum the instanton density is given by Eq. (14), where

$d_{\overline{\mathrm{MS}}}=\frac{2 e^{5 / 6}}{\pi^{2}\left(N_{c}-1\right) !\left(N_{c}-2\right) !} e^{-1.511374 N_{c}+0.291746 N_{f}} ;$

$g^{2}\left(\rho \Lambda_{\overline{\mathrm{MS}}}\right)$ is the running coupling constant at two loop order,

$$
g^{2}(x)=\frac{(4 \pi)^{2}}{\beta_{0} \log \left(x^{-2}\right)}\left(1-\frac{\beta_{1}}{\beta_{0}^{2}} \frac{\log \left(\log \left(x^{-2}\right)\right)}{\log \left(x^{-2}\right)}\right),
$$

with $\beta_{0}=\left(11 N_{c}-2 N_{f}\right) / 3$ and $\beta_{1}=34 N_{c}^{2} / 3-\left(13 N_{c} / 3-\right.$ $\left.1 / N_{c}\right) N_{f} \cdot \Lambda_{\overline{\mathrm{MS}}}$ is the renormalization mass scale of QCD in the modified minimal subtraction scheme. This expression is valid for small $x$, where $\log \left(x^{-2}\right)$ is positive. By asymptotic freedom, the coupling $g^{2}\left(\rho \Lambda_{\overline{\mathrm{MS}}}\right)$ is small at small $\rho$, so instantons are suppressed by the exponential of the classical action, $8 \pi^{2} / g^{2}$. Of necessity in a semiclassical computation, the exponential from the classical action dominates over the prefactor, $\sim g^{-12}$, which arises from the Jacobian for the collective coordinates of the instanton [4]. Conversely, when $\rho$ increases so does the coupling $g^{2}\left(\rho \Lambda_{\overline{\mathrm{MS}}}\right)$. The instanton density increases at first, but eventually decreases, suppressed by the prefactor from the Jacobian. The instanton density $n_{1}\left(\rho \Lambda_{\overline{\mathrm{MS}}}\right)$ is illustrated in Fig. (3); as seen there, there is a natural maximum when $\rho \sim 0.50 \Lambda_{\overline{\mathrm{MS}}}$ in the vacuum.

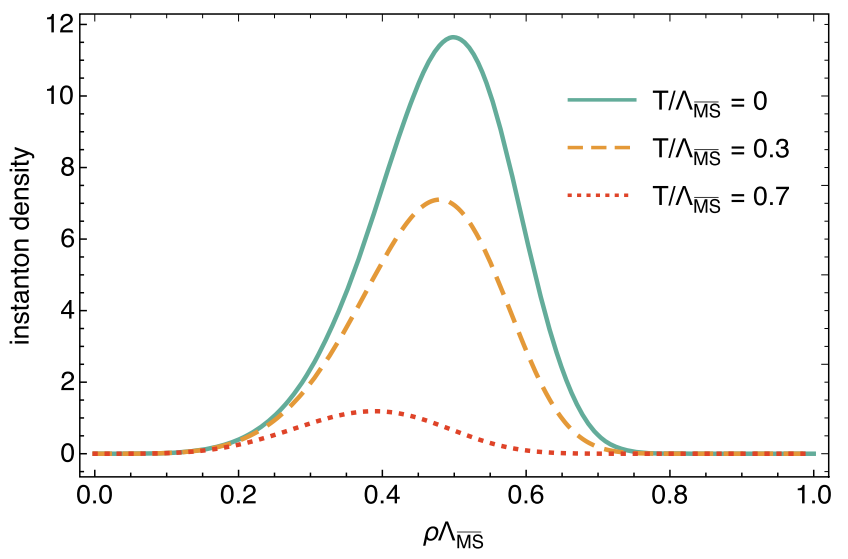

FIG. 3. The instanton density for a dilute instanton gas, Eq. (14), versus $\rho \Lambda_{\overline{\mathrm{MS}}}$, for two massless quarks and three different temperatures at $\mu=0$.

For a single instanton, at a temperature $T$ and quark chemical potential $\mu$, we approximate the change to the instanton density as

$$
\begin{aligned}
n_{1}(\rho, T, \mu)= & \exp \left\{-\frac{2 \pi^{2}}{g^{2}} \rho^{2} m_{D}^{2}-12 A(\pi \rho T)\right. \\
& \left.\times\left[1+\frac{1}{6}\left(N_{c}-N_{f}\right)\right]\right\} n_{1}(\rho),
\end{aligned}
$$

where

$$
m_{D}^{2}(T, \mu)=g^{2}\left[\left(\frac{N_{c}}{3}+\frac{N_{f}}{6}\right) T^{2}+\frac{N_{f}}{2 \pi^{2}} \mu^{2}\right]
$$

is the Debye mass at leading order, and $[12,13]$

$A(x)=-\frac{1}{12} \log \left(1+\frac{x^{2}}{3}\right)+.0129\left(1+\frac{0.159}{x^{3 / 2}}\right)^{-8}$.

The dominant term, $\sim \rho^{2} m_{D}^{2}$, is straightforward to understand. The topological charge is proportional to $\operatorname{tr}(\vec{E} \cdot \vec{B})$, where $\vec{E}$ and $\vec{B}$ are the color electric and magnetic fields. In any plasma, electrically charged particles screen static electric fields over distances $\sim 1 / m_{D}$. Since instantons must carry color electric fields, by itself Debye screening suffices to suppress the instanton density. Needless to say, this argument applies in a plasma where there is Debye screening, and not at low temperature.

For a single instanton at $T \neq 0$ and $\mu=0$, to one loop order the instanton density can be computed analytically either with puerile brute force [12] or by being clever [13]. The computation at $\mu \neq 0$ is, unexpectedly, rather more difficult $[15,21]$, and as of yet has not been computed for arbitrary values of $\rho \mu$. At nonzero $\mu$, then, we only include the leading contribution of quarks to the Debye mass. At $\mu=0$ and $T \neq 0$, though, numerically one can show that 
for the instanton density, the relative difference between the complete result and that with just the leading term from the Debye mass is small, at most a few percent for all values of $\rho T$. We comment that the instanton density to one loop order could be computed at $\mu \neq 0$ numerically using the Gelfand-Yaglom method [6], as has been done for the computation of the one loop determinant in an instanton field for quarks of nonzero mass [27].

Using the elementary ansatzes of Eqs. (14), (17) and (I3), we can calculate numerically how the density changes with temperature and chemical potential. Consider first $T \neq 0$ and $\mu=0$. As illustrated in the left plot of Fig. (4), as the Debye mass increases the instanton density decreases smoothly. To have some definite measure, we define the temperature as that where the integrated instanton density is $1 / 10^{\text {th }}$ its value at zero temperature as $T_{I}$. For three colors and two massless flavors, $T_{I}^{2 f l} \approx 0.71 \Lambda_{\overline{\mathrm{MS}}}$; for three massless flavors, $T_{I}^{3 f l} \approx 0.74 \Lambda_{\overline{\mathrm{MS}}}$. Using $\Lambda_{\overline{\mathrm{MS}}} \approx 332 \mathrm{MeV}$ [49], for two flavors $T_{I}^{2 f l} \approx 236 \mathrm{MeV}$, and $T_{I}^{3 f l} \approx 246 \mathrm{MeV}$ for three.

We stress that these numerical values are, at best, merely suggestive. Under our naive ansatz for a dilute instanton gas, the instanton density is very sensitive to the choice of $\Lambda_{\overline{\mathrm{MS}}}$; after all, merely on dimensional grounds the instanton density is $\sim\left(\Lambda_{\overline{\mathrm{MS}}}\right)^{4}$.

At nonzero temperature, to date the results from lattice QCD find that above temperatures $300-400 \mathrm{MeV}$, the fall off with temperature is a power law, whose value follows from the classical action for a single instanton and the running of the coupling $g^{2}$ with temperature. The overall prefactor measured in lattice QCD is approximately 10 times larger than the one loop result, but at high temperature perhaps this is ameliorated by the complete computation at two loop order [19]. It is still an open question as to whether topologically nontrivial fluctuations become dilute below [33,36] or above [34] the appropriate transition temperature. This is presumably due to a combination of

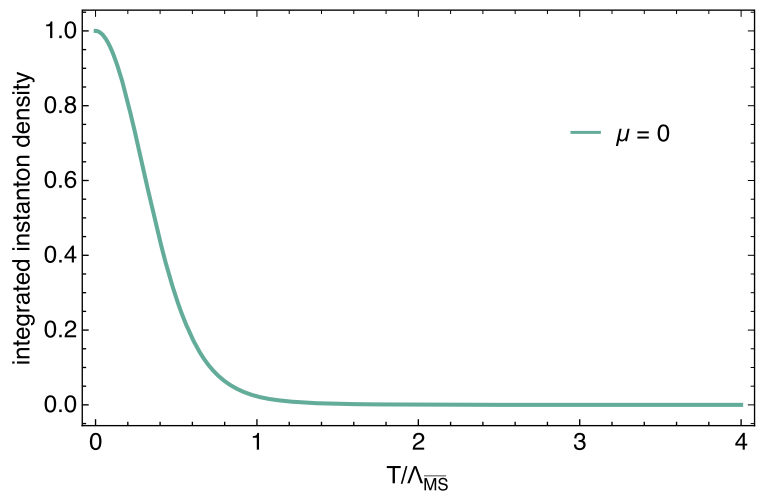

effects from fractional dyons and instantons with integral topological charge, either as a liquid or a gas. For our purposes, which is frankly phenomenological, the moral which we draw is that a dilute instanton gas is not a preposterous assumption, at least at temperatures about $T_{\chi}$.

Consider next the case of zero temperature and nonzero quark chemical potential. As for temperature, the density of instantons are smoothly suppressed as $\mu$ increases. The integrated density of instantons, shown in the right plot of Fig. 4 , is $1 / 10^{\text {th }}$ that in vacuum when $\mu_{I}^{2 f l} \approx 2.44 \Lambda_{\overline{\mathrm{MS}}}$ for two flavors, and $\mu_{I}^{3 f l} \approx 2.22 \Lambda_{\overline{\mathrm{MS}}}$ for three flavors. These correspond to $\mu_{I}^{2 f l} \approx 810 \mathrm{MeV}$ for two flavors, and $\mu_{I}^{3 f l} \approx$ $737 \mathrm{MeV}$ for three. Taking $T_{\chi} \approx 156 \mathrm{MeV}$ [50], this is approximately $\sim 1.5 \pi T_{\chi}$.

While even the instanton density at one loop order is incomplete at $\mu \neq 0$, we note that these are extremely high values of the quark chemical potential. They are almost into the perturbative regime, for $\mu>1 \mathrm{GeV}$ [54].

This gross disparity has a simple origin and thus may persist a more careful analysis. In a thermal bath, or the Fermi sea of cold, dense quarks, instantons are suppressed primarily because of Debye screening. As can be seen from the expression for the Debye mass in Eq. (I4), the natural scale for the chemical potential is $\mu \approx \pi T$. Indeed, as the Euclidean energy of any fermion field is an odd multiple of $\pi T$, this balance between $\mu$ and $\pi T$ is true of the propagator at tree level.

The weak dependence upon the quark chemical potential can also be understood in the limit of large $N_{c}$. As $N_{c} \rightarrow \infty$ the coupling $g^{2} \sim 1 / N_{c}$, so that if the number of quark flavors $N_{f}$ is held fixed as $N_{c} \rightarrow \infty$, any effects of quarks are suppressed by $\sim 1 / N_{c}$. In the plane of $T$ and $\mu$, large $N_{c}$ then generates a "quarkyonic" regime [55]. Our naive estimate for a dilute instanton gas is simply another illustration of this.

At present, numerical simulations of lattice QCD with classical computers can only provide results at nonzero

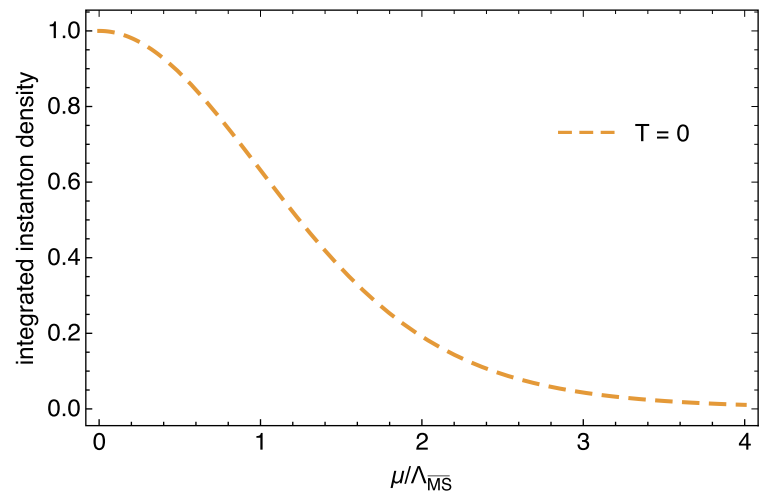

FIG. 4. For two flavors, the ratio of the density of an instanton gas, integrated over $\rho$ and normalized to the vacuum value. The left plot shows this as function of $T / \Lambda_{\overline{\mathrm{MS}}}$ at $\mu=0$ and the right plot as a function of $\mu / \Lambda_{\overline{\mathrm{MS}}}$ at $T=0$. This demonstrates graphically that instantons evaporate much more slowly as the quark chemical potential increases, as opposed to increasing the temperature. 
temperature and $\mu \leq T$. Simulations of cold, dense quark matter may be possible with quantum computers but will not be available for some time. Studying the dense regime of QCD with functional continuum methods, on the other hand, is possible even in the near-term [47]. Still, using an effective model, such as a dilute gas of instantons, is useful for developing the physical picture before results from first principles are available.
[1] S. L. Adler, Axial vector vertex in spinor electrodynamics, Phys. Rev. 177, 2426 (1969); J. S. Bell and R. Jackiw, A PCAC puzzle: $\pi^{0} \rightarrow \gamma \gamma$ in the $\sigma$ model, Nuovo Cimento A 60, 47 (1969); K. Fujikawa, Path Integral Measure for Gauge Invariant Fermion Theories, Phys. Rev. Lett. 42, 1195 (1979).

[2] A. A. Belavin, A. M. Polyakov, A. S. Schwartz, and Yu. S. Tyupkin, Pseudoparticle solutions of the Yang-Mills equations, Phys. Lett. 59B, 85 (1975).

[3] H. Fritzsch, M. Gell-Mann, and H. Leutwyler, Advantages of the color octet gluon picture, Phys. Lett. 47B, 365 (1973).

[4] G. 't Hooft, Symmetry Breaking through Bell-Jackiw Anomalies, Phys. Rev. Lett. 37, 8 (1976); Computation of the quantum effects due to a four-dimensional pseudoparticle, Phys. Rev. D 14, 3432 (1976); How instantons solve the U(1) problem, Phys. Rep. 142, 357 (1986).

[5] C. G. Callan, Jr., R. F. Dashen, and D. J. Gross, The structure of the gauge theory vacuum, Phys. Lett. 63B, 334 (1976); R. Jackiw and C. Rebbi, Vacuum Periodicity in a Yang-Mills Quantum Theory, Phys. Rev. Lett. 37, 172 (1976); C. G. Callan, Jr., R. F. Dashen, and D. J. Gross, Toward a theory of the strong interactions, Phys. Rev. D 17, 2717 (1978); A mechanism for quark confinement, Phys. Lett. 66B, 375 (1977); A theory of Hadronic structure, Phys. Rev. D 19, 1826 (1979); N. Andrei and D. J. Gross, The effect of instantons on the short distance structure of Hadronic currents, Phys. Rev. D 18, 468 (1978).

[6] S. R. Coleman, The uses of instantons, Subnucl. Ser. 15, 805 (1979).

[7] G. 't Hooft (to be published); E. Witten, Some Exact Multi -Instanton Solutions of Classical Yang-Mills Theory, Phys. Rev. Lett. 38, 121 (1977); R. Jackiw, C. Nohl, and C. Rebbi, Conformal properties of pseudoparticle configurations, Phys. Rev. D 15, 1642 (1977).

[8] M. F. Atiyah, N. J. Hitchin, V. G. Drinfeld, and Yu. I. Manin, Construction of instantons, Phys. Lett. 65A, 185 (1978).

[9] E. Corrigan, D. B. Fairlie, R. G. Yates, and P. Goddard, The construction of selfdual solutions to $\mathrm{SU}(2)$ gauge theory, Commun. Math. Phys. 58, 223 (1978).

[10] N. H. Christ, E. J. Weinberg, and N. K. Stanton, General selfdual Yang-Mills solutions, Phys. Rev. D 18, 2013 (1978); C. W. Bernard, Instanton interactions at the one loop level, Phys. Rev. D 18, 2026 (1978).

[11] B. Grossman, Zero energy solutions of the Dirac equation in an N-pseudoparticle field, Phys. Lett. 61A, 86 (1977); H. Osborn, Solutions of the Dirac equation for general instanton solutions, Nucl. Phys. B140, 45 (1978); E. Corrigan, D. B. Fairlie, S. Templeton, and P. Goddard, A Green's function for the general selfdual gauge field, Nucl. Phys.
B140, 31 (1978); E. Corrigan, P. Goddard, H. Osborn, and S. Templeton, Zeta function regularization and multiinstanton determinants, Nucl. Phys. B159, 469 (1979); P. Goddard, P. Mansfield, and H. Osborn, The functional measure for the exact SU(2) two instanton solution, Phys. Lett. 98B, 59 (1981); H. Osborn, Semiclassical functional integrals for selfdual gauge fields, Ann. Phys. (N.Y.) 135, 373 (1981).

[12] D. J. Gross, R. D. Pisarski, and L. G. Yaffe, QCD and instantons at finite temperature, Rev. Mod. Phys. 53, 43 (1981).

[13] C. P. Korthals Altes and A. Sastre, Thermal instanton determinant in compact form, Phys. Rev. D 90, 125002 (2014); Caloron correction to the effective potential in thermal gluodynamics, arXiv:1501.00474.

[14] M. A. Shifman, A. I. Vainshtein, and V. I. Zakharov, Instanton density in a theory with massless quarks, Nucl. Phys. B163, 46 (1980); A. I. Vainshtein, V. I. Zakharov, V. A. Novikov, and M. A. Shifman, ABC's of instantons, Sov. Phys. Usp. 25, 195 (1982); M. A. Shifman, A. I. Vainshtein, and V. I. Zakharov, Instantons in nonperturbative QCD vacuum, Nucl. Phys. B165, 45 (1980).

[15] C. Aragao de Carvalho, The instanton density in a quark gas, Nucl. Phys. B183, 182 (1981); V. Baluni, Screening of instantons in the quark gas, Phys. Lett. 106B, 491 (1981); M. Chemtob, Instanton effects in dense quark matter, Nucl. Phys. B184, 497 (1981).

[16] E. V. Shuryak, Suppression of instantons as the origin of confinement, Phys. Lett. 79B, 135 (1978); The role of instantons in quantum chromodynamics. 1. Physical vacuum, Nucl. Phys. B203, 93 (1982); The role of instantons in quantum chromodynamics. 2. Hadronic structure, Nucl. Phys. B203, 116 (1982); The role of instantons in quantum chromodynamics. 3. Quark-Gluon plasma, Nucl. Phys. B203, 140 (1982); Pseudoscalar mesons and instantons, Nucl. Phys. B214, 237 (1983); E. V. Shuryak and J. J. M. Verbaarschot, Mesonic correlation functions in the random instanton vacuum, Nucl. Phys. B410, 55 (1993); E.-M. Ilgenfritz and E. V. Shuryak, Chiral symmetry restoration at finite temperature in the instanton liquid, Nucl. Phys. B319, 511 (1989); Quark induced correlations between instantons drive the chiral phase transition, Phys. Lett. B 325, 263 (1994); T. Schäfer and E. V. Shuryak, The interacting instanton liquid in QCD at zero and finite temperature, Phys. Rev. D 53, 6522 (1996); Instantons in QCD, Rev. Mod. Phys. 70, 323 (1998); R. Rapp, T. Schäfer, E. V. Shuryak, and M. Velkovsky, Diquark Bose Condensates in High Density Matter and Instantons, Phys. Rev. Lett. 81, 53 (1998); High density QCD and instantons, 
Ann. Phys. (N.Y.) 280, 35 (2000); E. V. Shuryak and I. Zahed, Instanton induced effects in QCD high-energy scattering, Phys. Rev. D 62, 085014 (2000); T. Schäfer, Instantons and the large N(c) limit, A.D. 2004, in Large N(c) QCD 2004. Proceedings, International Workshop, Trento, Italy (2004), pp. 28-45, https://doi.org/10.1142/ 9789812701725_0003; J. Liao and E. Shuryak, Strongly coupled plasma with electric and magnetic charges, Phys. Rev. C 75, 054907 (2007); Electric flux tube in magnetic plasma, Phys. Rev. C 77, 064905 (2008); Y. Liu, E. Shuryak, and I. Zahed, Confining dyon-antidyon Coulomb liquid model. I., Phys. Rev. D 92, 085006 (2015); Light quarks in the screened dyon-antidyon Coulomb liquid model. II, Phys. Rev. D 92, 085007 (2015); Light adjoint quarks in the instanton-dyon liquid model IV, Phys. Rev. D 94, 105012 (2016); The instanton-dyon liquid model III: Finite chemical potential, Phys. Rev. D 94, 105011 (2016); The instanton-dyon liquid model V: Twisted light quarks, Phys. Rev. D 94, 105013 (2016); Dense instanton-dyon liquid Model: Diagrammatics, Phys. Rev. D 98, 014023 (2018); M. A. Lopez-Ruiz, Y. Jiang, and J. Liao, Confinement, holonomy and correlated instanton-dyon ensemble I: SU(2) Yang-Mills theory, Phys. Rev. D 97, 054026 (2018); Confinement from correlated instanton-dyon ensemble in SU(2) Yang-Mills theory, Phys. Rev. D 99, 114013 (2019); E. Shuryak, Light-front wave functions of mesons, baryons and pentaquarks, with topology-induced local 4-quark interaction, Phys. Rev. D 100, 114018 (2019).

[17] D. Diakonov and V. Yu. Petrov, Quark propagator and Chiral condensate in an instanton vacuum, Zh. Eksp. Teor. Fiz. 89, 361 (1985) [Sov. Phys. JETP 62, 204 (1985]; Instanton based vacuum from Feynman variational principle, Nucl. Phys. B245, 259 (1984); Chiral xondensate in the instanton vacuum, Phys. Lett. 147B, 351 (1984); A theory of light quarks in the instanton vacuum, Nucl. Phys. B272, 457 (1986); Meson current correlation functions in instanton vacuum, Sov. Phys. JETP 62, 431 (1985) [Zh. Eksp. Teor. Fiz. 89, 751 (1985)]; D. Diakonov, Instantons at work, Prog. Part. Nucl. Phys. 51, 173 (2003); D. Diakonov, N. Gromov, V. Petrov, and S. Slizovskiy, Quantum weights of dyons and of instantons with nontrivial holonomy, Phys. Rev. D 70, 036003 (2004); D. Diakonov and N. Gromov, SU(N) caloron measure and its relation to instantons, Phys. Rev. D 72, 025003 (2005); N. Gromov and S. Slizovskiy, Fermionic determinant for dyons and instantons with nontrivial holonomy, Phys. Rev. D 71, 125019 (2005); D. Diakonov and V. Petrov, Confining ensemble of dyons, Phys. Rev. D 76, 056001 (2007); F. Bruckmann, S. Dinter, E.-M. Ilgenfritz, M. Muller-Preussker, and M. Wagner, Cautionary remarks on the moduli space metric for multidyon simulations, Phys. Rev. D 79, 116007 (2009);

[18] D. Diakonov, Topology and confinement, Nucl. Phys. B, Proc. Suppl. 195, 5 (2009).

[19] T. R. Morris, D. A. Ross, and C. T. Sachrajda, Higher order quantum corrections in the presence of an instanton background field, Nucl. Phys. B255, 115 (1985); A. Ringwald and F. Schrempp, Confronting instanton perturbation theory with QCD lattice results, Phys. Lett. B 459, 249 (1999).
[20] N. Dorey, T. J. Hollowood, V. V. Khoze, and M. P. Mattis, The valculus of many instantons, Phys. Rep. 371, 231 (2002).

[21] T. Schäfer, Instantons and the chiral phase transition at nonzero baryon density, Phys. Rev. D 57, 3950 (1998); D. T. Son, M. A. Stephanov, and A. R. Zhitnitsky, Instanton interactions in dense matter QCD, Phys. Lett. B 510, 167 (2001); T. Schäfer, Instanton effects in QCD at high baryon density, Phys. Rev. D 65, 094033 (2002); QCD and the etaprime mass: Instantons or confinement? Phys. Rev. D 67, 074502 (2003); D. Toublan and A. R. Zhitnitsky, Confinement-deconfinement phase transition at nonzero chemical potential, Phys. Rev. D 73, 034009 (2006); T. Hatsuda, M. Tachibana, N. Yamamoto, and G. Baym, New Critical Point Induced by the Axial Anomaly in Dense QCD, Phys. Rev. Lett. 97, 122001 (2006); N. Yamamoto, Instanton-induced crossover in dense QCD, J. High Energy Phys. 12 (2008) 060; J.-W. Chen, K. Fukushima, H. Kohyama, K. Ohnishi, and U. Raha, U(A)(1) Anomaly in hot and dense QCD and the critical surface, Phys. Rev. D 80, 054012 (2009); N. Yamamoto and T. Kanazawa, Dense QCD in a Finite Volume, Phys. Rev. Lett. 103, 032001 (2009); T. Brauner, K. Fukushima, and Y. Hidaka, Two-color quark matter: U(1) (A) restoration, superfluidity, and quarkyonic phase, Phys. Rev. D 80, 074035 (2009); Erratum, 81, 119904 (2010); H. Abuki, G. Baym, T. Hatsuda, and N. Yamamoto, The NJL model of dense three-flavor matter with axial anomaly: The low temperature critical point and BEC-BCS diquark crossover, Phys. Rev. D 81, 125010 (2010); K. Fukushima and T. Hatsuda, The phase diagram of dense QCD, Rep. Prog. Phys. 74, 014001 (2011); M. Eto, M. Nitta, and N. Yamamoto, Confined monopoles induced by quantum effects in dense QCD, Phys. Rev. D 83, 085005 (2011); M. Mitter and B.-J. Schaefer, Fluctuations and the axial anomaly with three quark flavors, Phys. Rev. D 89, 054027 (2014); S. Raghavendra, V. C. Shastry, V. K. Nilakanthan, and K. B. Vijaya Kumar, The effect of confined one gluon exchange potential and instanton induced interaction in nucleon-nucleon interaction, Commun. Theor. Phys. 72, 065301 (2020); S.-I. Shim, A. Hosaka, and H.-C. Kim, Heavy baryon production with an instanton interaction, arXiv:1908.02966.

[22] J. M. Pawlowski, Exact flow equations and the U(1) problem, Phys. Rev. D 58, 045011 (1998); H. Gies and C. Wetterich, Universality of spontaneous chiral symmetry breaking in gauge theories, Phys. Rev. D 69, 025001 (2004); H. Gies, J. Jaeckel, J. M. Pawlowski, and C. Wetterich, Do instantons like a colorful background? Eur. Phys. J. C 49, 997 (2007); J. Braun, M. Leonhardt, and M. Pospiech, Fierz-complete NJL model study. II. Toward the fixed-point and phase structure of hot and dense two-flavor QCD, Phys. Rev. D 97, 076010 (2018); Fierz-complete NJL model study III: Emergence from quark-gluon dynamics, Phys. Rev. D 101, 036004 (2020).

[23] H. Reinhardt and R. Alkofer, Instanton induced flavor mixing in mesons, Phys. Lett. B 207, 482 (1988); A. A. Osipov and B. Hiller, Path integral bosonization of the 't Hooft determinant: Fluctuations and multiple vacua, Phys. Lett. B 539, 76 (2002). 
[24] D. U. Jungnickel and C. Wetterich, Effective action for the chiral quark-meson model, Phys. Rev. D 53, 5142 (1996); Effective linear meson model, Eur. Phys. J. C 1, 669 (1998); M. Grahl and D. H. Rischke, Functional renormalization group study of the two-flavor linear sigma model in the presence of the axial anomaly, Phys. Rev. D 88, 056014 (2013)

[25] M. Dine, G. Festuccia, L. Pack, and W. Wu, Reliable semiclassical computations in QCD, Phys. Rev. D 82, 065015 (2010); M. Dine, P. Draper, and G. Festuccia, Instanton effects in three flavor QCD, Phys. Rev. D 92, 054004 (2015); M. Dine, P. Draper, L. Stephenson-Haskins, and D. Xu, Axions, instantons, and the Lattice, Phys. Rev. D 96, 095001 (2017).

[26] M. Heller and M. Mitter, Pion and $\eta$-meson mass splitting at the two-flavour chiral crossover, Phys. Rev. D 94, 074002 (2016); G. Fejos, Functional dependence of axial anomaly via mesonic fluctuations in the three flavor linear sigma model, Phys. Rev. D 92, 036011 (2015); G. Fejos and A. Hosaka, Thermal properties and evolution of the $U_{A}(1)$ factor for $2+1$ flavors, Phys. Rev. D 94, 036005 (2016).

[27] G. V. Dunne, J. Hur, C. Lee, and H. Min, Precise Quark Mass Dependence of Instanton Determinant, Phys. Rev. Lett. 94, 072001 (2005); Calculation of QCD instanton determinant with arbitrary mass, Phys. Rev. D 71, 085019 (2005); G. V. Dunne, Connecting the chiral and heavy quark limits: Full mass dependence of fermion determinant in an instanton background, J. Phys. A 39, 6283 (2006).

[28] R. D. Pisarski and V. V. Skokov, How tetraquarks can generate a second chiral phase transition, Phys. Rev. D 94, 054008 (2016).

[29] F. Giacosa, A. Koenigstein, and R. D. Pisarski, How the axial anomaly controls flavor mixing among mesons, Phys. Rev. D 97, 091901 (2018).

[30] G. 't Hooft, Some twisted selfdual solutions for the YangMills equations on a hypertorus, Commun. Math. Phys. 81, 267 (1981); S. Sedlacek, A direct method for minimizing tYang-Mills functional over four manifolds, Commun. Math. Phys. 86, 515 (1982); P. van Baal, Some results for SU(N) gauge fields on the hypertorus, Commun. Math. Phys. 85, 529 (1982); K.-M. Lee and P. Yi, Monopoles and instantons on partially compactified D-branes, Phys. Rev. D 56, 3711 (1997); T. C. Kraan and P. van Baal, Monopole constituents inside SU(n) calorons, Phys. Lett. B 435, 389 (1998); Periodic instantons with nontrivial holonomy, Nucl. Phys. B533, 627 (1998); K.-M. Lee and C.-h. Lu, SU(2) calorons and magnetic monopoles, Phys. Rev. D 58, 025011 (1998); K.-M. Lee, Instantons and magnetic monopoles on $R^{3} * S^{1}$ with arbitrary simple gauge groups, Phys. Lett. B 426, 323 (1998); P. van Baal, QCD in a finite volume, in At the frontier of particle physics, edited by M. Shifman (2000), vol. 2, pp. 683-760; F. Bruckmann and P. van Baal, Multicaloron solutions, Nucl. Phys. B645, 105 (2002); F. Bruckmann, D. Nogradi, and P. van Baal, Constituent monopoles through the eyes of fermion zero modes, Nucl. Phys. B666, 197 (2003); Higher charge calorons with nontrivial holonomy, Nucl. Phys. B698, 233 (2004); E. Poppitz and $\mathrm{M}$. Unsal, Index theorem for topological excitations on $R^{3} * S^{1}$ and Chern-Simons theory, J. High Energy Phys. 03 (2009) 027.
[31] M. Unsal, Magnetic bion condensation: A new mechanism of confinement and mass gap in four dimensions, Phys. Rev. D 80, 065001 (2009); M. Shifman and M. Unsal, QCD-like theories on $\mathrm{R}(3) \times \mathrm{S}(1)$ : A smooth journey from small to large $\mathrm{r}(\mathrm{S}(1))$ with double-trace deformations, Phys. Rev. D 78, 065004 (2008); P. C. Argyres and M. Unsal, The semiclassical expansion and resurgence in gauge theories: New perturbative, instanton, bion, and renormalon effects, J. High Energy Phys. 08 (2012) 063; E. Poppitz, T. Schäfer, and M. Ünsal, Universal mechanism of (semi-classical) deconfinement and theta-dependence for all simple groups, J. High Energy Phys. 03 (2013) 087; G. Basar, G. V. Dunne, and M. Unsal, Resurgence theory, ghost-instantons, and analytic continuation of path integrals, J. High Energy Phys. 10 (2013) 041; G. V. Dunne and M. Unsal, Uniform WKB, Multi-instantons, and resurgent trans-series, Phys. Rev. D 89, 105009 (2014); G. V. Dunne and M. Ünsal, New nonperturbative methods in quantum field theory: From large-n orbifold equivalence to bions and resurgence, Annu. Rev. Nucl. Part. Sci. 66, 245 (2016); M. C. Ogilvie, Phases of gauge theories, J. Phys. A 45, 483001 (2012); Confinement on $R^{3} \times S^{1}$ : Continuum and lattice, Int. J. Mod. Phys. A 29, 1445003 (2014); K. Aitken, A. Cherman, and M. Ünsal, Vacuum structure of Yang-Mills theory as a function of $\theta$, J. High Energy Phys. 09 (2018) 030; T. Misumi, Y. Tanizaki, and M. Ünsal, Fractional $\theta$ angle, 't Hooft anomaly, and quantum instantons in charge- $q$ multi-flavor Schwinger model, J. High Energy Phys. 07 (2019) 018.

[32] K. Hashimoto and T. Izubuchi, Eta-prime meson from two flavor dynamical domain wall fermions, Prog. Theor. Phys. 119, 599 (2008); F. Bruckmann, E.-M. Ilgenfritz, B. Martemyanov, and B. Zhang, The vortex structure of SU(2) calorons, Phys. Rev. D 81, 074501 (2010); E. M. Ilgenfritz, B. V. Martemyanov, and M. Muller-Preussker, Cooling study of Dirac sheets in SU(3) lattice gauge theory below Tc, Phys. Rev. D 86, 074515 (2012); V. G. Bornyakov, E. M. Ilgenfritz, B. V. Martemyanov, V. K. Mitrjushkin, and M. Müller-Preussker, Topology across the finite temperature transition studied by overimproved cooling in gluodynamics and QCD, Phys. Rev. D 87, 114508 (2013); V. G. Bornyakov, E. M. Ilgenfritz, B. V. Martemyanov, and M. Muller-Preussker, Dyon structures in the deconfinement phase of lattice gluodynamics: Topological clusters, holonomies and Abelian monopoles, Phys. Rev. D 91, 074505 (2015); V. G. Bornyakov, E. M. Ilgenfritz, B. V. Martemyanov, and M. Müller-Preussker, Dyons near the transition temperature in lattice QCD, Phys. Rev. D 93, 074508 (2016); A. Di Giacomo and M. Hasegawa, Instantons and monopoles, Phys. Rev. D 91, 054512 (2015); J. Frison, R. Kitano, H. Matsufuru, S. Mori, and N. Yamada, Topological susceptibility at high temperature on the lattice, J. High Energy Phys. 09 (2016) 021; V. G. Bornyakov, E. M. Ilgenfritz, and B. V. Martemyanov, Dyons near the transition temperature in SU(3) lattice gluodynamics, J. Phys. G 45, 055006 (2018); E. Itou, Fractional instanton of the SU(3) gauge theory in weak coupling regime, J. High Energy Phys. 05 (2019) 093; L. Giusti and M. Lüscher, Topological susceptibility at $T>\mathrm{Tc}$ from master-field simulations of the SU(3) gauge theory, Eur. Phys. J. C 79, 207 (2019). 
[33] S. Aoki, H. Fukaya, and Y. Taniguchi, Chiral symmetry restoration, eigenvalue density of Dirac operator and axial U(1) anomaly at finite temperature, Phys. Rev. D 86, 114512 (2012); G. Cossu, S. Aoki, H. Fukaya, S. Hashimoto, T. Kaneko, H. Matsufuru, and J.-I. Noaki, Finite temperature study of the axial U(1) symmetry on the lattice with overlap fermion formulation, Phys. Rev. D 87, 114514 (2013); Erratum, 88, 019901 (2013); H. Fukaya, S. Aoki, G. Cossu, S. Hashimoto, T. Kaneko, and J. Noaki (JLQCD Collaboration), $\eta^{\prime}$ meson mass from topological charge density correlator in QCD, Phys. Rev. D 92, 111501 (2015); A. Tomiya, G. Cossu, S. Aoki, H. Fukaya, S. Hashimoto, T. Kaneko, and J. Noaki, Evidence of effective axial U(1) symmetry restoration at high temperature QCD, Phys. Rev. D 96, 034509 (2017); 96, 079902(A) (2017); S. Aoki, G. Cossu, H. Fukaya, S. Hashimoto, and T. Kaneko (JLQCD Collaboration), Topological susceptibility of QCD with dynamical Möbius domain-wall fermions, Prog. Theor. Exp. Phys. 2018, 043 B07 (2018).

[34] A. Bazavov et al. (HotQCD Collaboration), The chiral transition and $U(1)_{A}$ symmetry restoration from lattice QCD using domain wall fermions, Phys. Rev. D 86, 094503 (2012); M. I. Buchoff et al., QCD chiral transition, U(1)A symmetry and the dirac spectrum using domain wall fermions, Phys. Rev. D 89, 054514 (2014); V. Dick, F. Karsch, E. Laermann, S. Mukherjee, and S. Sharma, Microscopic origin of $U_{A}(1)$ symmetry violation in the high temperature phase of QCD, Phys. Rev. D 91, 094504 (2015); P. Petreczky, H.-P. Schadler, and S. Sharma, The topological susceptibility in finite temperature QCD and axion cosmology, Phys. Lett. B 762, 498 (2016).

[35] G. Grilli di Cortona, E. Hardy, J. Pardo Vega, and G. Villadoro, The QCD axion, precisely, J. High Energy Phys. 01 (2015) 034; C. Bonati, M. D’Elia, M. Mariti, G. Martinelli, M. Mesiti, F. Negro, F. Sanfilippo, and G. Villadoro, Axion phenomenology and $\theta$-dependence from $N_{f}=2+1$ lattice QCD, J. High Energy Phys. 03 (2016) 155; S. Borsanyi, M. Dierigl, Z. Fodor, S. D. Katz, S. W. Mages, D. Nogradi, J. Redondo, A. Ringwald, and K. K. Szabo, Axion cosmology, lattice QCD and the dilute instanton gas, Phys. Lett. B 752, 175 (2016); S. Borsanyi et al., Calculation of the axion mass based on high-temperature lattice quantum chromodynamics, Nature (London) 539, 69 (2016).

[36] B. B. Brandt, A. Francis, H. B. Meyer, O. Philipsen, D. Robaina, and $\mathrm{H}$. Wittig, On the strength of the $U_{A}(1)$ anomaly at the chiral phase transition in $N_{f}=2$ QCD, J. High Energy Phys. 12 (2016) 158.

[37] P. T. Jahn, G. D. Moore, and D. Robaina, $\chi_{\text {top }}\left(T \gg T_{\mathrm{c}}\right)$ in pure-glue QCD through reweighting, Phys. Rev. D 98, 054512 (2018); Improved reweighting for QCD topology at high temperature, arXiv:2002.01153.

[38] E. Vicari and H. Panagopoulos, Theta dependence of SU(N) gauge theories in the presence of a topological term, Phys. Rep. 470, 93 (2009).

[39] C. Bonati, M. D'Elia, H. Panagopoulos, and E. Vicari, Change of $\theta$ Dependence in 4D SU(N) Gauge Theories Across the Deconfinement Transition, Phys. Rev. Lett. 110, 252003 (2013).
[40] E. Witten, Instantons, the quark model, and the $1 / \mathrm{n}$ expansion, Nucl. Phys. B149, 285 (1979); Current algebra theorems for the U(1) Goldstone Boson, Nucl. Phys. B156, 269 (1979); E. WittenLarge N Chiral dynamics, Ann. Phys. (N.Y.) 128, 363 (1980); A. D’ Adda, P. Di Vecchia, and M. Luscher, Confinement and Chiral symmetry breaking in $\mathrm{CP}^{\mathrm{n}-1}$ models with quarks, Nucl. Phys. B152, 125 (1979); P. Nath and R. L. Arnowitt, The U(1) problem: Current algebra and the theta vacuum, Phys. Rev. D 23, 473 (1981); C. Rosenzweig, J. Schechter, and C. G. Trahern, Is the effective Lagrangian for QCD a Sigma model?, Phys. Rev. D 21, 3388 (1980); P. Di Vecchia, F. Nicodemi, R. Pettorino, and G. Veneziano, Large n, Chiral approach to pseudoscalar masses, mixings and decays, Nucl. Phys. B181, 318 (1981); P. Di Vecchia and G. Veneziano, Chiral dynamics in the large n limit, Nucl. Phys. B171, 253 (1980).

[41] B. Lucini and M. Panero, SU(N) gauge theories at large N, Phys. Rep. 526, 93 (2013); Introductory lectures to large- $N$ QCD phenomenology and lattice results, Prog. Part. Nucl. Phys. 75, 1 (2014).

[42] It is understood that all determinants are normalized with the corresponding determinant for a vanishing gluon background field.

[43] C. W. Bernard, Gauge zero modes, instanton determinants, and QCD calculations, Phys. Rev. D 19, 3013 (1979).

[44] To be precise, this configuration is given by $\rho_{i} \ll\left|x_{f i}-z_{1}\right| \ll\left|x_{f i}-z_{2}\right| \approx\left|R_{12}\right|$. For other source locations $x_{f i}$, corrections to the non-local interaction are generated. Eq. (8) is asymmetric in $\rho_{1}$ and $\rho_{2}$ because the interaction is generated by zero modes close to instanton 1. The symmetry is restored by including an equal contribution from zero modes which are close to instanton 2 . See Eqs. (E3) and (E4), and (F3) and (F4), of the Appendix.

[45] There are further interactions which while color singlet overall, are products of operators that are not. These are relevant for color superconductivity, and are ignored here.

[46] There is one additional anomalous quartic term, $\left(\operatorname{Tr} \Phi^{\dagger} \Phi\right)\left(\operatorname{det} \Phi+\operatorname{det} \Phi^{\dagger}\right)$, which is induced by instantons with $Q=1$. A detailed analysis shows that, while it changes the numerical values of the couplings, the behavior of the masses as a function of the reduced temperature does not change. For details, see Appendix $\mathrm{H}$.

[47] M. Mitter, J. M. Pawlowski, and N. Strodthoff, Chiral symmetry breaking in continuum QCD, Phys. Rev. D 91, 054035 (2015); J. Braun, L. Fister, J. M. Pawlowski, and F. Rennecke, From quarks and gluons to Hadrons: Chiral symmetry breaking in dynamical QCD, Phys. Rev. D 94, 034016 (2016); A. K. Cyrol, M. Mitter, J. M. Pawlowski, and N. Strodthoff, Nonperturbative quark, gluon, and meson correlators of unquenched QCD, Phys. Rev. D 97, 054006 (2018); C. S. Fischer, QCD at finite temperature and chemical potential from DysonSchwinger equations, Prog. Part. Nucl. Phys. 105, 1 (2019); W.-j. Fu, J. M. Pawlowski, and F. Rennecke, QCD phase structure at finite temperature and density, Phys. Rev. D 101, 054032 (2020).

[48] A. Boccaletti and D. Nogradi, The semi-classical approximation at high temperature revisited, J. High Energy Phys. 03 (2020) 045.

[49] M. Tanabashi et al. (Particle Data Group), Review of particle physics, Phys. Rev. D 98, 030001 (2018). 
[50] Y. Aoki, G. Endrodi, Z. Fodor, S. D. Katz, and K. K. Szabo, The order of the quantum chromodynamics transition predicted by the standard model of particle physics, Nature (London) 443, 675 (2006); Y. Aoki, Z. Fodor, S. D. Katz, and K. K. Szabo, The QCD transition temperature: Results with physical masses in the continuum limit, Phys. Lett. B 643, 46 (2006); Y. Aoki, S. Borsanyi, S. Durr, Z. Fodor, S. D. Katz, S. Krieg, and K. K. Szabo, The QCD transition temperature: Results with physical masses in the continuum limit II, J. High Energy Phys. 06 (2009) 088; A. Bazavov et al., The chiral and deconfinement aspects of the QCD transition, Phys. Rev. D 85, 054503 (2012); S. Borsanyi, G. Endrodi, Z. Fodor, S. D. Katz, and K. K. Szabo, Precision $\mathrm{SU}(3)$ lattice thermodynamics for a large temperature range, J. High Energy Phys. 07 (2012) 056; A. Bazavov et al. (HotQCD Collaboration), Equation of state in $(2+1)$-flavor QCD, Phys. Rev. D 90, 094503 (2014); T. Bhattacharya et al., QCD Phase Transition with Chiral Quarks and Physical Quark Masses, Phys. Rev. Lett. 113, 082001 (2014).

[51] F. Rennecke, Higher topological charge and the QCD vacuum, arXiv:2003.13876.

[52] M. F. Atiyah and I. M. Singer, The index of elliptic operators on compact manifolds, Bull. Am. Math. Soc. 69, 422 (1963).

[53] M. Creutz, On invariant integration over $\mathrm{Su}(\mathrm{N})$, J. Math. Phys. (N.Y.) 19, 2043 (1978).
[54] A. Kurkela, P. Romatschke, and A. Vuorinen, Cold quark matter, Phys. Rev. D 81, 105021 (2010); I. Ghisoiu, T. Gorda, A. Kurkela, P. Romatschke, M. Säppi, and A. Vuorinen, On high-order perturbative calculations at finite density, Nucl. Phys. B915, 102 (2017); T. Gorda, A. Kurkela, P. Romatschke, M. Säppi, and A. Vuorinen, Nextto-Next-to-Next-to-Leading Order Pressure of Cold Quark Matter: Leading Logarithm, Phys. Rev. Lett. 121, 202701 (2018).

[55] L. McLerran and R. D. Pisarski, Phases of cold, dense quarks at large N(c), Nucl. Phys. A796, 83 (2007); L. McLerran, K. Redlich, and C. Sasaki, Quarkyonic matter and Chiral symmetry breaking, Nucl. Phys. A824, 86 (2009); A. Andronic et al., Hadron production in ultrarelativistic nuclear collisions: Quarkyonic matter and a triple point in the phase diagram of QCD, Nucl. Phys. A837, 65 (2010); T. Kojo, Y. Hidaka, L. McLerran, and R. D. Pisarski, Quarkyonic Chiral spirals, Nucl. Phys. A843, 37 (2010); T. Kojo, Y. Hidaka, K. Fukushima, L. D. McLerran, and R. D. Pisarski, Interweaving Chiral spirals, Nucl. Phys. A875, 94 (2012); R. D. Pisarski, V. V. Skokov, and A. M. Tsvelik, Fluctuations in cool quark matter and the phase diagram of quantum chromodynamics, Phys. Rev. D 99, 074025 (2019); L. McLerran and S. Reddy, Quarkyonic Matter and Neutron Stars, Phys. Rev. Lett. 122, 122701 (2019); R. D. Pisarski, V. V. Skokov, and A. Tsvelik, A pedagogical introduction to the Lifshitz Regime, Universe 5, 48 (2019). 